\title{
Questions and Answers in an Orthoalgebraic Approach
}

\author{
Reinhard Blutner
}

Published online: 31 January 2012

(C) The Author(s) 2012. This article is published with open access at Springerlink.com

\begin{abstract}
Taking the lead from orthodox quantum theory, I will introduce a handy generalization of the Boolean approach to propositions and questions: the orthoalgebraic framework. I will demonstrate that this formalism relates to a formal theory of questions (or 'observables' in the physicist's jargon). This theory allows formulating attitude questions, which normally are non-commuting, i.e., the ordering of the questions affects the answer behavior of attitude questions. Further, it allows the expression of conditional questions such as "If Mary reads the book, will she recommend it to Peter?", and thus gives the framework the semantic power of raising issues and being informative at the same time. In the case of commuting observables, there are close similarities between the orthoalgebraic approach to questions and the Jäger/Hulstijn approach to question semantics. However, there are also differences between the two approaches even in case of commuting observables. The main difference is that the Jäger/Hulstijn approach relates to a partition theory of questions whereas the orthoalgebraic approach relates to a 'decorated' partition theory (i.e. the elements of the partition are decorated by certain semantic values). Surprisingly, the orthoalgebraic approach is able to overcome most of the difficulties of the Jäger/Hulstijn approach. Furthermore, the general approach is suitable to describe the different types of (non-commutative) attitude questions as investigated in modern survey research. Concluding, I will suggest that an active dialogue between the traditional model-theoretic approaches to semantics and the orthoalgebraic paradigm is mandatory.
\end{abstract}

R. Blutner $(\bowtie)$

Institute for Logic, Language, and Computation, University of Amsterdam, Amsterdam,

The Netherlands

e-mail: blutner@uva.nl 
Keywords Attitude questions · Commutativity · Conditional questions ·

Decorated partitions · Orthoalgebra · Orthodox quantum theory · Qubit .

Question semantics · Structured propositions · Survey research

\section{Introduction}

In modern survey research (e.g. Schuman and Presser 1981; Tourangeau et al. 2000) a distinction is made between factual questions and attitude questions. In a factual question the interviewer typically asks the respondent about her personal activities or circumstances. In attitude questions, by contrast, the interviewer seeks the respondent's opinion about an issue.

(1) a. What is your name?

b. Where do you live?

c. In what year did you first have an episode of back pain that lasted longer than a week?

(2) a. Do you think the use of marijuana should be made legal, or not?

b. Would you be for or against sex education in the public schools?

c. On the average, (Blacks/African-Americans) have worse jobs, income, and housing than white people. Do you think these differences are mainly due to discrimination ${ }^{1}$

For factual questions of the kind illustrated in (1), autobiographical memory forms a basic part of the required knowledge. To answer the questions, information is seldom retrieved from the direct experience of some facts; instead, it is mostly inferred. Attitude items, in contrast, rarely refer to any well-defined set of generally acceptable facts. If there is a set of facts that determine the accuracy of answers to questions like (2a), these are presumably about the respondent's beliefs or attitudes. The subjective character of attitudes makes it difficult, if not impossible, to verify survey reports about them. Moreover, the respondent may have a number of beliefs about marijuana and other illicit drugs - some of them contradictory. Hence, it is unclear which beliefs the respondent could reasonably consider in framing an answer to (2a). Because of this open-ended character, it does not make much sense even to discuss the accuracy of answers to questions like those listed in (2).

So far as I can see, logical-semantic analyses of questions have almost exclusively concentrated on factual questions. Despite their practical importance attitude questions have been widely ignored in this literature. Presently, I cannot see that anybody has made any real connection between modern survey research and the semantics of questions, although there are interesting empirical findings which cry for a logicalsemantic analysis. For example, survey researchers have demonstrated repeatedly that the same question often produces quite different answers, depending on the question context (for numerous survey examples, see Schuman and Presser 1981; Sudman and Bradburn 1982). To cite just one particularly well-documented example, a group of (North-American) subjects were asked whether "the United States should let Communist reporters come in here and send back to their papers the news as they see

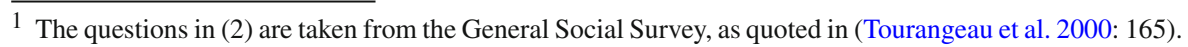


it?" The other group was asked whether "a Communist country like Russia should let American newspaper reporters come in and send back to their papers the news as they see it?" Support for free access for the Communist reporters varied sharply according to whether that question preceded or followed the question on American reporters. ${ }^{2}$

The example illustrates the non-commutative character of attitude questions. This contrasts with factual questions, which normally do not show these effects. The noncommutative character of questions invites to consider the analogy between the treatment of questions in natural language semantics and the treatment of observables in modern physics. An observable is a question addressing nature (Where is the particle? What is its momentum? How many particles are in this domain?). And a measurement is the process of answering such questions. In classical physics, the ordering of the observables does not matter: observables commute. However, this is not the case if one considers observables that relate to properties of the micro world, such as the place and the momentum of photons, or the place and the energy of electrons. Heisenberg's main motivation for developing his theory (which was later called matrix mechanics) concerned the non-commutativity of the observables under discussion. Heisenberg's famous principle of uncertainty is a direct consequence of the fact that the order of observables can matter. Investigating the close mathematical analogy between order effects for attitude questions and order effects for physical observables is one important concern of the present article.

There is another formal aspect that connects the semantic analysis of questions to the mathematical analysis of observables. It concerns the partition of the state space that underlies the analysis of questions and observables. Consider, for example, the semantics of questions as developed by Groenendijk and Stokhof $(1984,1997)$ (abbreviated to GS). It is assumed that the state space is formed by a set of possible worlds which constitute the basis for building propositions and other semantic objects. Further, it is assumed that a question partitions the state space in equivalent classes where two states are equivalent if they give the same answer. This picture is not unlike the formal treatment of observables in quantum physics (e.g. Birkhoff and von Neumann 1936; Von Neumann 1932). In this case the state space is formed by a vector space and it is assumed that an observable partitions the state space in equivalent classes. Two states are seen as equivalent if they give the same result when measuring the observable.

Quantum physics reduces to classical physics if all observables are commuting. In this case, possible worlds can be identified with an (orthonormal) base of vectors hulling the whole vector space. It could be supposed that in this special case the treatment of observables and questions coincides in quantum physics and partition semantics. However this is not the case. The main reason is that in quantum theory the partitions are decorated (by the eigenvalues of the corresponding eigenspaces). The GS partition semantics does not know any decorations. Hence, questions such as (3a) and

\footnotetext{
2 The differences are quite dramatic: in a study of 1950, 36\% accepted communist reporters when the communist question came first and $73 \%$ accepted them when the question came second. When the study was repeated in 1982 , the numbers changed to 55 vs. $75 \%$.
} 
(3b) are considered equivalent assuming that 'open' and 'not closed' are semantically equivalent. ${ }^{3}$
(3) a. Is the door open?
b. Is the door closed?
c. Peter knows if the door is open.
d. Peter knows if the door is closed.

Consequently, the equivalence of (3c) and (3d) comes out automatically as a result of the equivalence between ( $3 a)$ and ( $3 b)$. Though not doubting the equivalence between (3c) and (3d) there are some doubts about the semantic equivalence of the two questions (3a) and (3b). The partition theory cannot handle the difference between the term-answers 'yes' and 'no'. "The reason is that the two propositions in the question meaning cannot be identified as 'positive' and 'negative', as one proposition is just the complement of the other-recall that all we have is sets of possible worlds, not their descriptions." (Krifka 2001: 290). ${ }^{4}$

Another difference between the two treatments concerns the analysis of conditional questions, illustrated by the following example ${ }^{5}$ :

(4) If electron 1 has spin $\uparrow$ what is the spin of electron 2?

Using the operator formalism of quantum theory, it is not difficult to formalize the semantic content of (4). However, the standard GS partition theory does not introduce a conditional operator general enough to express conditional questions. To be sure, the GS partition theory makes a strict distinction between questions and answers. Semantically, questions are described by an equivalence relation and answers are described by propositions (sets of possible worlds). Surprisingly, such a distinction is not made in quantum physics where both questions (observables) and answers (projection spaces) can be treated as particular linear operators. It is exactly this uniform treatment that allows a straightforward treatment of conditional questions. Recently, Gerhard Jäger and Joris Hulstijn have proposed an extension of the GS question theory that is able to handle conditional questions. We will call this approach the Jäger/Hulstijn (JH) approach (Hulstijn 1997; Jäger 1996). In their analysis of conditional questions a new operator is designed which will deserve our critical attention.

In the present paper, I follow three aims. Firstly, I will discuss the similarities and differences between the question theories developed by students of formal semantics and those developed independently (and much earlier) by physicists. In order to do this in a methodologically sound way, I have to restrict myself to the classical case of commuting observables/questions. This is the scenario where the framework of

\footnotetext{
${ }^{3}$ If you do not like the examples with 'open' and 'closed' because you feel the relevant constructions are not really semantically equivalent, you can construct similar examples using 'even' and 'odd'.

${ }^{4}$ Another point is that the partition theory cannot analyze the difference between "Is the door open?" and "Is the door open or not?" Both questions are analyzed by assuming the same partition. However, in the first case a possible answer could be 'no', but not in the second. Of course, partition semantics could claim a pragmatic approach for handling this problem. However, I do not see how this could work in a systematic way without arbitrary assumptions.

5 The construction of such examples is very common in literature which interprets the famous EPR thought experiment (cf. Einstein et al. 1935).
} 
quantum theory reduces to a classical, Boolean framework. Secondly, mainly based on the commuting case, I will propose a new solution to unify the analysis of questions and answers based on the operator formalism of quantum theory. I will demonstrate that this analysis reduces to a decorated partition theory of questions if question order effects are excluded. This solution bears a close resemblance to the JH theory. However, there are also some important differences, and I will demonstrate how several shortcomings of the $\mathrm{JH}$ approach can be overcome by using the decorated partition theory derived from the operator formalism known from quantum theory. The third aim is to give up the feature of order-independence (commutativity) of questions/observables and to develop a framework which describes the different kinds of order effects found in attitude questions. The theory is applied to attitude questions in the context of opinion forming as investigated in survey research and personality psychology.

The organization of this paper is as follows. In the next section, I give a concise introduction to question semantics as developed in the JH approach. Section 3 introduces the basic orthoalgebraic framework as it has been used in modern quantum theory. It further gives a concise introduction into the basic elements of linear algebra which are indispensable for understanding this paper. In Sect. 4, I develop the orthoalgebraic semantics for questions and answers. The model deviates in some respects from the question semantics treated in Sect. 3; both the differences and the strict similarities are discussed. A comparison with alternative approaches is made in Sect. 5. Further, I will point out the close similarity between the so-called structured meaning approach and the present theory of decorated partitions. Section 6 extends the approach to include attitude questions and describing question order effects. Section 7 , finally, draws some general conclusions.

\section{The Jäger/Hulstijn Approach to Question Semantics}

Many recent analyses of the meaning of questions start with three assumptions: (i) to understand a question is to understand what counts as an answer to that question; (ii) an answer to a question is an assertion or a statement; (iii) an assertion is identical with its propositional content (cf. Groenendijk and Stokhof 1997, p. 1066). Different approaches that fit into this scheme are (a) Hamblin (1973) who identifies a question with the set of propositional contents of its possible answers, (b) Karttunen (1977) for whom it is the smaller set of its true answers, and (c) the GS partition theory (Groenendijk and Stokhof 1984, 1997) which defines the meaning of a question as the set of its complete answers. Krifka (2001) categorises these theories under the label proposition set approach and contrasts it with the so-called structured meaning approach (Hausser 1983; Loeser 1968; Tichy 1978; Von Stechow 1991). ${ }^{6}$ In the latter, the answers to $w h$-questions are identified with the senses of noun phrases rather than

\footnotetext{
6 Besides the proposition set theory and the structured meaning approach there are other approaches that fit into the basic scheme, for instance Nelken's and Francez' bilattice approach to the semantics of questions (Nelken and Francez 1999, 2000, 2002) or Wiśniewski's interrogative semantics based on erotetic inferences (Wiśniewski 1995). For space reasons we cannot discuss these approaches in the present paper which focuses on discussing variants of the partition-theoretic models and their relationship to the treatment of observables in quantum mechanics.
} 
of sentences. Accordingly, the meanings of questions are constructed as functions that yield a proposition when applied to the semantic value of the answer (see Sect. 5 for more discussion).

Question semantics is a version of update semantics which takes into account that sentences do not only provide data, but also raise issues. In the GS theory these two tasks are strictly divided over two syntactic categories: declarative sentences provide data and interrogative sentences raise issues. This strategy has its limitations. For instance, it does not allow us to represent conditional questions. ${ }^{7}$ Recent developments of question semantics deviate from the classical picture in different ways. Some writers claim it is sufficient to modify classical partition theory in order to adapt it for the purposes of conditional questions (Hulstijn 1997; Jäger 1996). Others claim classical partition semantics has to be given up for the same purpose (Groenendijk 2009; Roelofsen and van Gool 2010; Velissaratou 2000).

Obviously, the simplest way to unify questions and answers is to adapt partition semantics by saying that not the whole domain of possible worlds has to be partitioned but only a subpart of it. A proposition then can be seen as partitioning the set of all worlds that make the proposition true into a partition consisting just of one element: the set of worlds that make the proposition true. A conditional question then partitions the set of all worlds where the antecedent of the conditional is true. As we will see immediately, such a version conforms to the JH approach. Surprisingly, this is the variant of partition theory that most naturally results from the orthoalgebraic approach as used in quantum theory.

Let us introduce the language of the formal setting first. Consider the logical query language $Q L$ to be the language of propositional logic $L$, extended with a question operator "?" and a (non-standard) conditional operator " $\Rightarrow$ ". Formally, $Q L$ can be defined as the smallest set containing $L$ and satisfying the following two clauses:

$$
\begin{aligned}
& \text { a. if } \varphi \in Q L \text { then } ? \varphi \in Q L \\
& \text { b. if } \varphi, \psi \in Q L \text { then }(\varphi \Rightarrow \psi) \in Q L,(\neg \varphi) \in Q L, \text { and }(\varphi \wedge \psi) \in Q L
\end{aligned}
$$

Following the JH approach (Hulstijn 1997; Jäger 1996) we can formulate the following semantic update clauses where $W$ is a set of possible worlds, $\omega$ is a classical interpretation function (assigning subsets of $W$ to the atomic propositional symbols), and $\sigma$ is an information state modeled by an equivalence relation over the logical space, $\sigma \subseteq$ $W^{2}$. What is defined in this recursive way is the information change potential $\ll \xi \gg$ of any expression $\xi$ of $Q L$. It is a function from information states to information states.

$$
\begin{array}{ll}
\text { a. } & \sigma \ll p \gg=\sigma \cap\left\{(u, v) \in W^{2}: u \in \omega(p) \text { and } v \in \omega(p)\right\} \\
\text { b. } & \sigma \ll \varphi \gg=\sigma \cap\left\{(u, v) \in W^{2}:(u, u) \notin \sigma \ll \varphi \gg \text { and }(v, v) \notin \sigma \ll \varphi \gg\right\} \\
\text { c. } & \sigma \ll \varphi \wedge \psi \gg=\sigma \ll \varphi \gg \ll \psi \gg \\
\text { d. } & \sigma \ll ? \varphi \gg=\{(u, v) \in \sigma:(u, u) \in \sigma \ll \varphi \gg \text { iff }(v, v) \in \sigma \ll \varphi \gg\} \\
\text { e. } & \sigma \ll \varphi \Rightarrow \psi \gg=\{(u, v) \in \sigma \ll ? \varphi \gg \text { : if }(u, v) \in \sigma \ll \varphi \gg \text { then }(u, v) \in \\
& \sigma \ll \varphi \gg \ll \psi \gg\}
\end{array}
$$

\footnotetext{
7 There are two other potential shortcomings, but discussing them goes beyond the scope of the present paper: (i) a proper treatment of hybrid expressions such as disjunctions which act as questions and assertions; (ii) the account for certain typological facts that demand a unification of question and declarative semantics (cf. Groenendijk 2008).
} 
Fig. 1 Picture of meaning $p$ (assertion) in question semantics

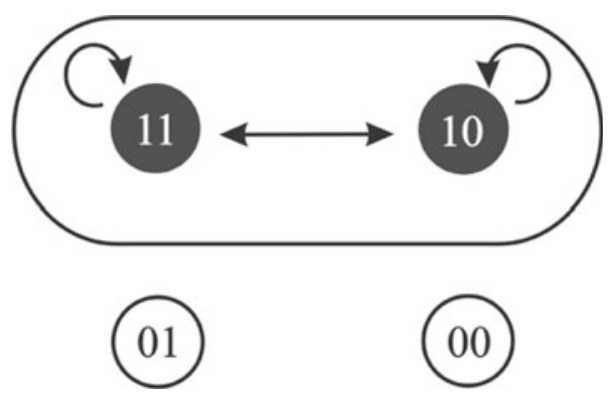

For atomic formulas $p$, the first clause expresses the elimination of all possibilities incompatible with $p$. Negation is modelled in (6b) by set complement. The use of the intersection operator in the definition makes sure that negation is a so-called declarative update (cf. Hulstijn 1997). In (6c) conjunction is modeled by function composition on updates leading to a sequential notion of conjunction. The definition (6d) defines question by equivalent relations where two worlds are considered equivalent if they give the same answer to question ? $\varphi$.

In the JH framework, the standard definition for $\vee$ is used: $\varphi \vee \psi \equiv \neg(\neg \varphi \wedge \neg \psi)$. In order to model conditional questions the standard implication $\varphi \rightarrow \psi \equiv(\varphi \wedge \neg \psi)$ cannot be used. The reason is that the clause for negation is declarative, i.e. no structure can be induced under the scope of negation. But conditional questions give an interesting structure and for this reason $\mathrm{JH}$ have proposed an alternative definition for conditionals $\Rightarrow$, as shown in definition (6e). In this definition, the restriction $(u, v) \in \sigma \ll ? \varphi \gg$ is required. That means the antecedent of the conditional must become an issue. Leaving out this restriction it would no longer be guaranteed that the result is an equivalence relation (cf. Hulstijn 1997, footnote 10).

By way of illustration, let us consider a fragment with two atoms $p$ and $q$. Identifying possible worlds with functions assigning the truth values 1 (true) and 0 (false) to the atoms, we get four possible worlds abbreviated by 10, 11, 01, 00. Interpreting atoms by sets of worlds in which the atoms are true gives the obvious assignments $\omega(p)=\{10,11\}$ and $\omega(q)=\{01,11\}$. Figure 1 shows the meaning of $p$ in question semantics assuming an initial information state $\sigma=W^{2}$ (representing a logically empty state, a tautology if you want). Consequently, we are concerned with a single equivalence class that captures the logical space of $p$ (set of worlds that make $p$ true).

Figure 2 pictures the meaning of ? $p$ in question semantics. It is constituted by two equivalence classes which partition the space $W$ of possible worlds.

The meaning of the conditional interrogative $p \Rightarrow ? q$ is pictured in Fig. 3. It is the partition of the logical space consisting of three blocks. The blocks of the partition correspond to the propositions expressed by $p \wedge q, p \wedge \neg q$ and $\neg p$.

There is a controversy about this result, mainly concentrated on examples of the following kind (Velissaratou 2000):

(7) A: If Mary reads this book, will she recommend it to Peter?

B: Mary does not read this book. 
Fig. 2 Picture of meaning ? $p$ in question semantics

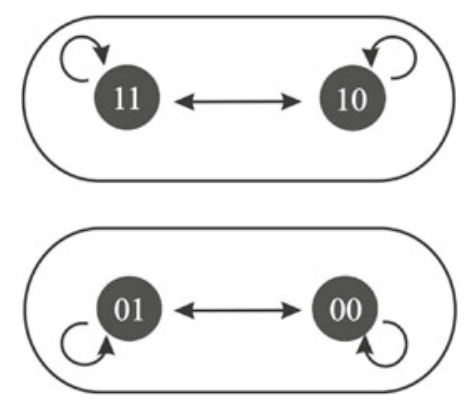

Fig. 3 Picture of meaning $p \Rightarrow ? q$ in question semantics

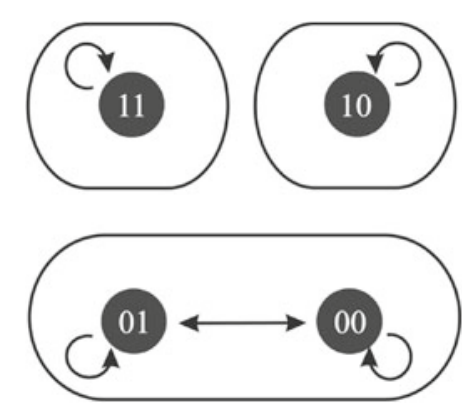

According to the JH approach the answer given by (7B) should count as a (complete) answer, having the same status as the two other possible answers, namely "yes, he will" and "no, he won't". ${ }^{8}$ However, there is a problem with this analysis. As pointed out by Isaacs and Rawlins (2008), responses like (7B) are not answers in the technical sense; i.e., they do not resolve the issue raised by the question. Instead, they indicate a species of presupposition failure. To say it in another way, the question in (7A) is about whether Mary will recommend the book to Peter. Denying the antecedent addresses the ground on which the question stands, not the question itself. The partition semantics taken by JH fails to give any indication of the different status of the three blocks of the partition. There are further problems with this approach, to mention only one: $p \Rightarrow ? p$ comes out as semantically equivalent with $? p$, which is rather counter-intuitive.

In conclusion, we have seen some conceptual and empirical problems of the $\mathrm{JH}$ approach. The conceptual flaws are mainly related to the need of two different definitions of conditionals, one relating to the usual material implication, the other to the interrogative conditional. The empirical problems are due to the uniformity of the classical partition semantics, which gives all blocks of the partition the same status.

\section{Basic Concepts of Linear Algebra}

In this chapter, I will give a concise introduction to the parts of linear algebra that are required to understand the orthoalgebraic approach to semantics. At its centre we find

\footnotetext{
8 This possibility was also suggested by (Groenendijk and Stokhof 1997, fn. 29).
} 
Fig. 4 Geometric representation of complex numbers in a two-dimensional plane

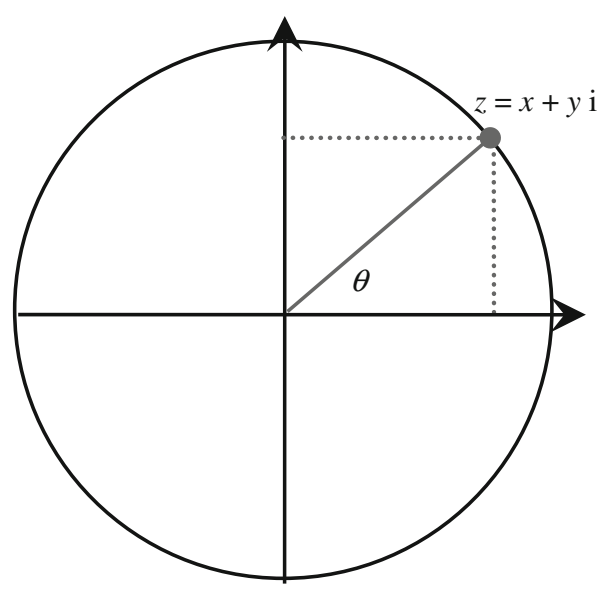

the concept of a Hilbert space $\mathscr{H}$, i.e. a vector space upon which an inner product (= scalar product) is defined and which makes use of complex numbers instead of real ones. Further, we consider linear operators defined on $\mathscr{H}$, and we discuss the spectral decomposition of a particular class of linear operators. The decomposition takes place in terms of so-called projection operators which project certain subspaces of $\mathscr{H}$. The algebraic structure underlying these projection operators is an orthoalgebra. In orthoalgebraic semantics, propositions are modeled by projection operators (or, equivalently, subspaces of $\mathscr{H}$ ).

\subsection{Complex Numbers and Quaternions}

Complex numbers can be conceptualized as pairs of real numbers. The first part of a complex number is called its real part; the second part is called its imaginary part. Complex numbers are usually written in the form $z=x+y \mathrm{i}$, with real numbers $x$ and $y$. The first part of this decomposition is called the real part of $z$, the latter part is called the imaginary part of $z$. For calculating with complex numbers the same rules can be used as for calculating with real numbers, respecting the assumption $\mathrm{i}^{2}=-1$. Complex numbers were introduced to allow for solutions of certain equations, such as $z^{2}+1=0$, that have no real solution (since the square of $z$ is 0 or positive, so $z^{2}+1$ cannot be zero). It is easy to see that the equation has two complex solutions: $z= \pm \mathrm{i}$. Generalizing the result, in 1799 Gauss published the first proof that an $n$th degree equation (which can be written as $z^{n}+a_{1} z^{n-1}+\cdots+a_{n-2} z^{2}+a_{n-1} z+a_{n}=0$ ) has $n$ roots each of the form $z=x+y$ i, for some real numbers $x$ and $y$. form

Through the Euler formula, a complex number $z=x+y$ i may be written in the

(8) $z=|z|(\cos \theta+i \sin \theta)=|z| e^{i \theta}$, where $|z|=\sqrt{x^{2}+y^{2}}$ and $\theta=\tan ^{-1}\left(\frac{y}{x}\right)$.

Figure 4 shows how complex numbers can be pictured in a two-dimensional plane by the use of the Euler formula (8). 
Each complex number $z=x+y$ i has a complex conjugate, written $z^{*}$ and defined by $z *=x-y$ i. The product $z z^{*}$ equals $|z|^{2}=x^{2}+y^{2}$.

\subsection{Vector Spaces}

I assume that the reader already has an intuitive idea about vectors. If not, look at a concrete example of a two-dimensional vector space consisting of all points in a plane represented by all ordered pairs $(x, y)$ of real numbers (you can visualize a vector by an arrow directed from the origin $(0,0)$ to the point $(x, y)$ in our Cartesian coordinate system. Simple examples for vectors are physical forces acting on mass points. Another class of examples is the oscillation of mass points described by functions of time, such as $\mathrm{h}(t)=a \cdot \cos (2 \pi f \cdot t)$, where the parameter $a$ is the amplitude of the oscillation and $f$ its frequency.

Intuitively, two physical forces can be superposed and the same idea of superposition applies for two physical oscillations. Formally, the crucial operation is vector addition. Another operation for vectors is scalar multiplication. Applying scalar multiplication changes the length of the vector but typically not its direction (only if the scalar is negative, the opposite direction is taken). In the case of oscillations, scalar multiplication does not account for a change of the amplitude only; it can also change the phase of the oscillation if the scalar is a complex number. For example, using the Euler formula (8), we can describe an oscillation with amplitude $a$ and frequency $f$ by the complex function $a \cdot \mathrm{e}^{i 2 \pi f \cdot t}$. Its real part then gives us the ordinary description $\mathrm{h}(t)=a \cdot \cos (2 \pi f \cdot t)$. Now let us multiply the complex function with the complex number $\mathrm{i}$. Because of the equivalence $\mathrm{i}=\mathrm{e}^{i \pi / 2}$ (Euler formula), we get the function $a \cdot \mathrm{e}^{i 2 \pi f \cdot(t+1 / 4 f)}$. This function describes the original oscillation phase shifted by $1 / 4$ of its period. Generally, by multiplying with a complex number $\mathrm{e}^{i \theta}$, we can describe a phase shift of $\theta / 2 \pi f$.

Vector spaces (denoted by $U, V, \ldots$ ) are sets of vectors that are closed under the two operations of vector addition and scalar multiplication. In other words, if all the vectors $u_{\mathrm{i}}(\mathrm{i}=1, \ldots, \mathrm{n})$ are elements of a vector space, then each linear combination of it, i.e. each sum $x_{1} \cdot u_{1}+x_{2} \cdot u_{2}+\cdots+x_{n} \cdot u_{n}$, is also an element of the vector space. Importantly, the two operations addition and scalar multiplication are subject to some simple conditions. We will not explain all the relevant conditions in detail because of space limitations. The addition operation has to satisfy commutativity, associativity, the existence of a null-element and of an additive inverse. Further, with regard to scalar multiplication the distributive properties are assumed and we have the multiplicative identity $1 \cdot u=u .{ }^{9}$ Vector spaces based on scalar multiplication with real numbers $\mathbf{R}$ are called real vector spaces; vector spaces based on scalar multiplication with complex numbers $\mathbf{C}$ are called complex vector spaces.

A subset of a vector space $U$ is called a subspace of $U$ if it is a vector space (i.e. closed under addition and scalar multiplication). Let $U_{1}$ and $U_{2}$ be two subspaces of $U$,

\footnotetext{
9 There are many good textbooks which introduce all the mathematical details. I refer the reader to Axler (1996) or Strang (2003). Advanced readers could likewise consider introductions into quantum information science, e.g. Vedral (2006).
} 
then the sum of the two vector spaces, written $U_{1}+U_{2}$, is the set of all possible sums of elements of $U_{1}$ and $U_{2}$. The sum of two vector spaces is a vector space again. We will say that the sum $U_{1}+U_{2}$ is a direct sum of the two subspaces $U_{1}$ and $U_{2}$ if and only if each element of the sum can be written uniquely as a sum $u_{1}+u_{2}$ where $u_{1} \in U_{1}$ and $u_{2} \in U_{2}$. It can be proven (e.g. Axler 1996 ) that a sum $U_{1}+U_{2}$ is a direct sum iff $U_{1} \cap U_{2}=\{0\}$. The linear hull of a list of vectors $\left(u_{1}, u_{2}, \ldots, u_{n}\right)$ in $U$ is defined as the set of all linear combinations of these vectors, denoted $\mathrm{LH}\left(u_{1}, u_{2}, \ldots, u_{n}\right)$. A vector space is called finitely dimensional if it is the linear hull of some finite list of vectors.

An important idea is the linear independence of a set of vectors. A set of vectors is called linearly independent if none of its elements is a linear combination of the others. Otherwise it is called linearly dependent. A basis of a vector space $U$ is a list of linearly independent vectors in $U$ iff $U$ is the linear hull of these vectors. If there are several bases of a vector space, it can be proven that the number of the vectors is the same in each base. This number is called the dimension of the vector space. Observe that $\operatorname{dim}\left(U_{1}+U_{2}\right)=\operatorname{dim}\left(U_{1}\right)+\operatorname{dim}\left(U_{2}\right)-\operatorname{dim}\left(U_{1} \cap U_{2}\right)$. This result allows the following conclusion: the dimension of the direct sum of two vector spaces is the sum of the dimensions of the two spaces.

Finally, we will consider the simple example of a two-dimensional, real vector space $\mathbf{R}^{2}=\left\{\left(x_{1}, x_{2}\right): x_{1} \in \mathbf{R}\right.$ and $\left.x_{2} \in \mathbf{R}\right\}$. Two subspaces of $\mathbf{R}^{2}$ are $\mathbf{R}^{1}{ }_{1}=\left\{\left(x_{1}, 0\right): x_{1} \in \mathbf{R}\right\}$ and $\mathbf{R}^{1}=\left\{\left(0, x_{2}\right): x_{2} \in \mathbf{R}\right\}$. Now we can write $\mathbf{R}^{2}=\mathbf{R}^{1}{ }_{1}+\mathbf{R}^{1}{ }_{2}$. This sum is a direct sum since $\mathbf{R}^{1}{ }_{1} \cap \mathbf{R}^{1}{ }_{2}=\{(0,0)\}$. The vector space $\mathbf{R}^{2}$ is the linear hull of the two vectors $(1,0)$ and $(0,1)$. An alternate base consists of the two vectors $(1,0)$ and $(1,1)$. The two vectors $(1,0)$ and $(0,1)$ are called orthogonal, but the two vectors $(1,0)$ and $(1,1)$ are not orthogonal. The notion of orthogonality can be made precise in terms of scalar product, a notion we introduce now.

\subsection{Scalar Product}

Scalar multiplication and scalar product are different operations. The former is an operation between (real or complex) numbers and vectors resulting in vectors; the latter is an operation between two vectors resulting in a (real or complex) number expressing the similarity of the two vectors. The scalar product of two vectors $u, v$ in a given vector space is written in the form $u \cdot v .^{10}$

If we take the example of the real vector space $\mathbf{R}^{2}$, the scalar product of two vectors $u=\left(x_{1}, x_{2}\right)$ and $v=\left(y_{1}, y_{2}\right)$ can be written as $u \cdot v=x_{1} y_{2}+x_{1} y_{2}$. The length of the vector $u$, also called the norm of $u$, results as $\|u\|=\sqrt{u^{2}}=\sqrt{x_{1}^{2}+x_{2}^{2}} \cdot{ }^{11}$ We can also write the scalar product of $u$ and $v$ in the form $u \cdot v=\|u\|\|v\| \cos \varphi$, where $\varphi$ is the angle between $u$ and $v$. Using this example, we can see some properties of the scalar product: (a) positivity: $u \cdot u \geq 0$ for all $u \in U$; (b) definiteness: $u \cdot u=0$ iff $u=0$,

\footnotetext{
10 The dot symbol ' $'$ ' is used both for the scalar product and scalar multiplication. This should not be confusing because the context always makes clear what is meant. Further, note that the dot symbol is usually omitted for scalar multiplication but not for the scalar product.

11 Generally, including the complex case the definition is $\|u\|=\sqrt{u u *}$.
} 
(c) additivity: $\left(u_{1}+u_{2}\right) \cdot u=u_{1} \cdot u+u_{2} \cdot u$, (d) homogeneity: $(\mathrm{a} u) \cdot v=a(u \cdot v)$ with a $\in \mathbf{R}$, (e) symmetry: $u \cdot v=v \cdot u$.

Usually, the scalar product is introduced in an axiomatic way. In this formulation exactly the axioms (a)-(d) are assumed. The symmetry property (e) is replaced by the axiom of conjugate symmetry: $u \cdot v=(v \cdot u) *$ (taking the case of complex numbers into account). Generally, two (non-zero) vectors are called orthogonal if their scalar product is zero. A list of vectors is called orthogonal if the vectors in the list are pairwise orthogonal; the list is called orthonormal if, in addition, all the vectors of the list have the unit norm 1. Ortho-gonal and orthonormal lists of vectors are always linearly independent and form a base of a vector space.

Finite vector spaces augmented with a scalar product are called (finite) Hilbert spaces, usually designated by $\mathscr{H}$. Hilbert spaces can be assumed to be hulled by a finite orthonormal base $\mathbf{S}=\left(u_{1}, u_{2}, \ldots, u_{n}\right)$ and each vector $u$ can be represented by a linear combination $x_{1} \cdot u_{1}+x_{2} \cdot u_{2}+\cdots+x_{n} \cdot u_{n}$ of the base vectors. The list $\left(x_{1}, x_{2}, \ldots, x_{n}\right)$ of the corresponding (complex) numbers in the linear combination representing $u$ is called its component vector (relative to base $\mathbf{S}$ ).

Let us assume that $U$ is a subspace of a Hilbert space $\mathscr{H}$. With the help of the scalar product the orthocomplement of $U$-written $U^{\perp}$ - can be defined as the set of vectors that are orthogonal to each vector in $U$ :

$$
U^{\perp}=\{u \in \mathscr{H}: u \cdot v=0 \text { for any } v \in U\}
$$

It is not difficult to prove that the orthocomplement is a vector space again and that $\mathscr{H}$ is the direct sum of $U$ and $U^{\perp}: \mathscr{H}=U+U^{\perp}$. In Sect. 3.5 we will consider the algebra that arises from considering the three basic operations on vector spaces: intersection, sum, and orthocomplement.

\subsection{Linear Operators}

Linear algebra is basically the study of linear operators on finite Hilbert spaces $\mathscr{H}$. A linear operator is a function a that maps $\mathscr{H}$ onto itself and which has the following properties: (a) additivity: $\mathbf{a}(u+v)=\mathbf{a} u+\mathbf{a} v$ for all $u, v \in \mathscr{H}$, (b) homogeneity: $\mathbf{a}(x u)=x(\mathbf{a} u)$ for all $u \in \mathscr{H}$ and all (real/complex) numbers $x$. With regard to an orthonormal base $\mathbf{S}=\left(u_{1}, u_{2}, \ldots, u_{n}\right)$ of a finite Hilbert space $\mathscr{H}$ we can assign a matrix to each linear operator. This matrix determines the linear operator uniquely. It is defined as follows:

$$
\mathbf{a}_{i j}=u_{\mathrm{i}} \cdot\left(\mathbf{a} u_{j}\right)
$$

Taking a vector $u$ with its components $\left(x_{1}, x_{2}, \ldots, x_{n}\right)$ we can determine the components $\left(y_{1}, y_{2}, \ldots, y_{n}\right)$ of the vector $v$ resulting from applying $\mathbf{a}$ to $u(v=\mathbf{a} u)$ by matrix multiplication:

$$
y_{i}=\sum_{j} \mathrm{a}_{i j} x_{j}
$$

In the following, we will concentrate on a special class of linear operators called normal operators. A linear operator a in $\mathscr{H}$ is called normal iff it satisfies the following condition: 
(12) $\mathbf{a} * \mathbf{a}=\mathbf{a a}^{*}$

Hereby the adjoint of $\mathbf{a}$, denoted $\mathbf{a}^{*}$, is defined by the following clause:

$(\mathbf{a} v) \cdot u=v \cdot(\mathbf{a} * u)$ for all vectors $u, v$ in $\mathscr{H}^{12}$

Linear operators can be characterized by their invariant subspaces. A vector space $U$ (subspace of $\mathscr{H}$ ) is called invariant under the transformation a iff for any $u \in U$ it holds that $\mathbf{a} u \in U$. An example of an invariant subspace of a linear operator $\mathbf{a}$ is the kernel or null space of a. It is defined as the set of vectors that project to zero; i.e. null $\mathbf{a}=\{u \in \mathscr{H}: \mathbf{a} u=0\}$. Using matrices to represent linear operators I will consider the following example: $\mathbf{a}=\left(\begin{array}{ll}1 & 2 \\ 2 & 4\end{array}\right)$. In this case, the kernel is spanned by the vector $\left(\begin{array}{l}-2 \\ 1\end{array}\right)$. This is easily checked by the following calculation: $\left(\begin{array}{ll}1 & 2 \\ 2 & 4\end{array}\right)\left(\begin{array}{l}-2 \\ 1\end{array}\right)=\left(\begin{array}{l}0 \\ 0\end{array}\right)$. Hence, we get null $\mathbf{a}=\left\{x\left(\begin{array}{l}-2 \\ 1\end{array}\right): x \in \mathrm{C}\right\}$.

Besides the kernel of an operator, there is another important class of invariant subspaces. These are the subspaces hulled by all eigenvectors of a linear operator a with a fixed eigenvalue $\lambda$. These subspaces are called eigenspaces of $\mathbf{a}$. The eigenspace of a for an eigenvalue $\lambda$ is defined as the set of all vectors $u$ satisfying the eigenvalue equation:

(14) $\mathbf{a} u=\lambda u$

The eigenspace of $\mathbf{a}$ for the eigenvalue $\lambda$ can be expressed as the kernel of the operator $\mathbf{a}-\lambda \mathbf{I}$, where $\mathbf{I}$ is the identity operator in $\mathscr{H}, \mathbf{I} u=u$ for all $u$ in $\mathscr{H}$.

Next we can introduce the central idea of linear algebra: spectral decomposition. Roughly, the idea is to decompose the operator into a system of invariant subspaces of the operator. The invariant subspaces are the eigenspaces of the operator considering the whole spectrum of its eigenvalues. To express the idea in a precise manner we have to introduce the concept of a projection operator. Intuitively, a projection operator in $\mathscr{H}$ projects all vectors of $\mathscr{H}$ onto one of $\mathscr{H}$ 's subspaces, say $U$. The direct sum $\mathscr{H}=U+U^{\perp}$ tells us that each vector $v$ of $\mathscr{H}$ can be uniquely represented as $v=u+u^{\prime}$ where $u \in U$ and $u^{\prime} \in U^{\perp}$. This leads us to the following definition of a projection operator $\mathbf{a}_{U}$ (projecting each vector of $\mathscr{H}$ into the $U$ ):

(15) $\mathbf{a}_{U}(v)=u$, where $v=u+u^{\prime}$ and $u \in U, u^{\prime} \in U^{\perp}$

Figure 5 gives a geometric illustration of a projection operator projecting a threedimensional real Hilbert space into a two-dimensional subspace.

Projection operators can be characterized by the property that their eigenvalues are 1 or 0 . An equivalent definition states that they are idempotent, i.e. $\mathbf{a a}=\mathbf{a}$.

By the use of the mathematics developed so far it can be proven that each normal operator has a spectral decomposition. That is, any normal operator a can be written in the following form:

(16) $\mathbf{a}=\sum_{\mathrm{i}} \lambda_{\mathrm{i}} \mathbf{a}_{\mathrm{i}}$, where $\mathbf{a}_{\mathrm{i}}$ denotes a projection operator that projects any vector of $\mathscr{H}$ into the eigenspace of a with eigenvalue $\lambda_{\mathrm{i}}$. The projection operators $\mathbf{a}_{\mathrm{i}}$ have distinct eigen-values, i.e. if $\mathrm{i} \neq \mathrm{j}$ then $\lambda_{\mathrm{i}} \neq \lambda_{\mathrm{j}}$.

\footnotetext{
12 In matrix representation, the adjoint is the conjugate transposed matrix; i.e. $\left(\mathbf{a}^{*}\right)_{\mathrm{ij}}=\mathbf{a}_{\mathrm{ji}}{ }^{*}$.
} 
Fig. 5 Illustration of a projection operator projecting $v \in \mathbf{R}^{3}$ into $u \in \mathbf{R}^{2}$

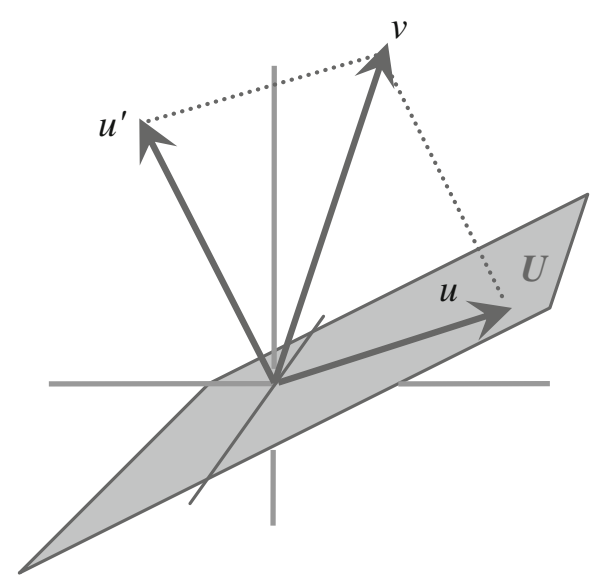

In the spectral decomposition of a normal operator $\mathbf{a}$, the projection operators $\mathbf{a}_{\mathrm{i}}$ are weighted with their eigenvalues $\lambda_{i}$. The operators $\mathbf{a}_{i}$ project the vector space $\mathscr{H}$ into the corresponding eigenspaces with eigenvalues $\lambda_{\mathrm{i}}$. These eigenspaces are pairwise orthogonal and their (direct) sum gives the full vector space $\mathscr{H}$. Another way of looking at the spectral decomposition (16) is by considering an orthonormal system of eigenvectors of a. This system hulls the whole vector space $\mathscr{H}$. Obviously, this system is partitioned into subsystems of base vectors corresponding to their eigenvalues. In other words, the spectral decomposition (16) can be seen as generating a decorated partition of the system of eigenvectors of $\mathbf{a}$.

In quantum mechanics, physical observables are represented by linear operators in a Hilbert space $\mathscr{H}$ exhibiting real eigenvalues. Such operators with real eigenvalues are called Hermitian operators. They always satisfy the condition $\mathbf{a}^{*}=\mathbf{a}$. Projection operators are always Hermitian. They represent observables that detect whether a certain vector projects into a specified subspace (eigenvalue 1) or not (eigenvalue 0 ). Using the spectral theorem, each Hermitian operator a can be decomposed into a sum of projection operators weighted by real numbers $\lambda_{\mathrm{i}}$ (their eigenvalues): $\mathbf{a}=\sum_{\mathrm{i}} \lambda_{i} \mathbf{a}_{\mathrm{i}}$.

Next, I have to explain the idea of a physical measurement, where the observable that is measured is represented by a Hermitian operator a. Assume that the considered physical system is in a certain state $u \in \mathscr{H}$. There are two possibilities now. First, the state $u$ is an eigenstate of $\mathbf{a}$, say with eigenvalue $\lambda_{\mathrm{i}}$. Then the measurement results in this eigenvalue and the state after the measurement is not changed (i.e. it is $u$ again). The second possibility is that the state $u$ is not an eigenstate of $\mathbf{a}$. In this case, quantum mechanics assumes that the act of measuring changes this state into another state which is always an element of one of the eigenspaces of a. It is decided by chance which eigenspace it is. There is no way to formulate a deterministic mechanism for this decision. Hence, indeterminism is an essential component of quantum mechanics. The only thing that can be predicted is the probability of finding the output state in a certain eigenspace of $\mathbf{a}$, say the space described by the projection operator $\mathbf{a}_{i}$. There is a simple rule to calculate this probability (called the Born rule): square the length of the projection $\mathbf{a}_{\mathrm{i}} u$, i.e. calculate $\left\|\mathbf{a}_{\mathrm{i}} u\right\|^{2}$; the result gives the probability that the state 
$u$ collapses in the eigenspace described by $\mathbf{a}_{\mathrm{i}}$. In other words, it gives the probability that the eigenvalue $\lambda_{i}$ is measured.

To repeat the deep insight from quantum theory: the act of measuring can change the state of the system. Only if the initial state is an eigenstate of the observable that is measured the final state is not changed by the measurement. Generally, a measurement can be seen as a question addressed to nature. The act of questioning can change the state of the system. This is not really surprising when considering modern versions of update semantics (such as the JH question semantics considered in Sect. 2). What really is puzzling, however, is the kind of ordering effects we predict for attitude questions. This phenomenon will be discussed in Sect. 6. In Sect. 4 we will consider the "classical case" of non-commuting observables/questions. Exploiting the analogy between observables in quantum theory and questions in update semantics, I will develop a new version of question semantics. In particular, I will show that most shortcomings of the $\mathrm{JH}$ approach can be resolved by using the underlying idea of decorated partitions.

\subsection{Pauli (Spin) Matrices}

In order to illustrate the concepts introduced so far, some simple examples are useful. Let us first consider the real vector space $\mathbf{R}^{2}$. I consider two operators $\sigma_{x}$ and $\sigma_{z}{ }^{13}$ which are given by the following matrices:

$$
\sigma_{x}=\left(\begin{array}{ll}
0 & 1 \\
1 & 0
\end{array}\right), \quad \sigma_{z}=\left(\begin{array}{ll}
1 & 0 \\
0 & -1
\end{array}\right)
$$

It is easy to check that the operator $\sigma_{z}$ has two orthogonal eigenvectors: the eigenvector $z^{+}=\left(\begin{array}{l}1 \\ 0\end{array}\right)$ with eigenvalue +1 and the eigenvector $z^{-}=\left(\begin{array}{l}0 \\ 1\end{array}\right)$ with eigenvalue -1 . The operator $\sigma_{x}$, in contrast, has the following two (normalized) eigenvectors: $x^{+}=\frac{1}{\sqrt{2}}\left(\begin{array}{l}1 \\ 1\end{array}\right)$ with eigenvalue +1 and $x^{-}=\frac{1}{\sqrt{2}}\left(\begin{array}{l}1 \\ -1\end{array}\right)$ with eigenvalue -1 . It is a simple exercise to verify the following spectral decompositions of the operators $\sigma_{x}$ and $\sigma z$, respectively:

$$
\begin{array}{ll}
\text { a. } & \sigma_{x}=\left(\begin{array}{ll}
0 & 1 \\
1 & 0
\end{array}\right)=\left(\begin{array}{ll}
\frac{1}{2} & \frac{1}{2} \\
\frac{1}{2} & \frac{1}{2}
\end{array}\right)+\left(\begin{array}{ll}
-\frac{1}{2} & \frac{1}{2} \\
\frac{1}{2} & -\frac{1}{2}
\end{array}\right) \\
\text { b. } & \sigma_{z}=\left(\begin{array}{ll}
1 & 0 \\
0 & -1
\end{array}\right)=\left(\begin{array}{ll}
1 & 0 \\
0 & 0
\end{array}\right)+\left(\begin{array}{ll}
0 & 0 \\
0 & -1
\end{array}\right)
\end{array}
$$

Figure 6 presents the corresponding geometric representations of the eigenstates of the operators $\sigma_{x}$ and $\sigma_{z}$. It is convenient to symbolize the two projection operators appearing in the decomposition of $\sigma_{z}$ by $\mathbf{1}$ and $\mathbf{0}$, where the operator $\mathbf{1}$ projects into the eigenvector $z^{+}$of $\sigma_{z}$ and the projection operator $\mathbf{0}$ projects into the

\footnotetext{
13 The term spin matrices and the special names $\sigma_{x}$ and $\sigma_{z}$ will be explained later when we consider the complex Hilbert space.
} 

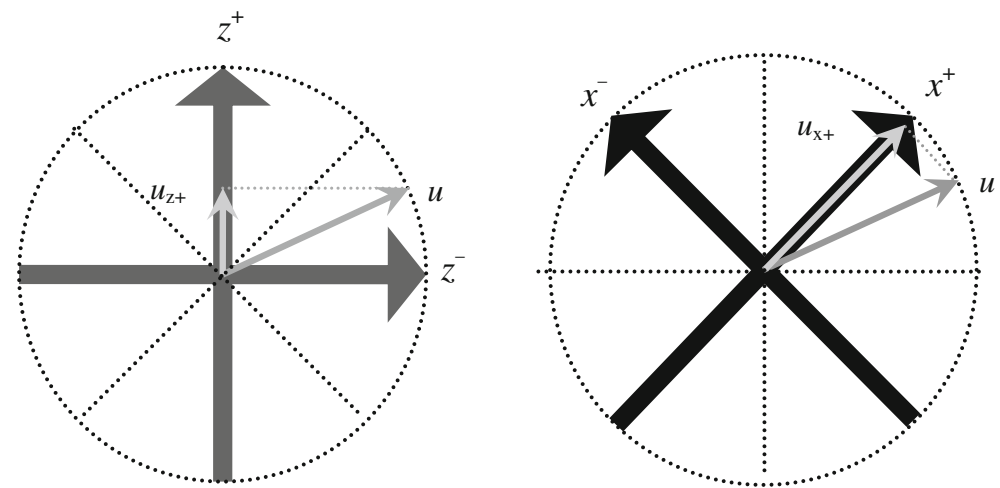

Fig. 6 Visual representation of the eigenstates of the operators $\sigma_{x}$ (right) and $\sigma_{z}$ (left). The vector $u$ is projected into the one-dimensional eigenspaces hulled by the vectors $x^{+}$(right) and $z^{+}$(left), respectively. The projections result in the vectors $u_{x+}$ and $u_{z+}$. The squares of the length of the vectors $u_{x+}$ and $u_{z+}$ are the probabilities of the yes-answers to the 'questions' $\sigma_{x}$ and $\sigma_{z}$, respectively

eigenvector $z^{-14}$ The operator $\mathbf{1}$ is different from the identity operator $\mathbf{I}$ introduced earlier. It is easy to check that $\mathbf{I}=\mathbf{0}+\mathbf{1}$. Note that $\sigma_{z}=\mathbf{1}-\mathbf{0}$. Further, note that $\mathbf{0}$ is different from the zero operator $\emptyset$ which maps each state of $\mathscr{H}$ to zero $(\emptyset u=0)$.

Now consider the state $u=\left(\begin{array}{l}0 \\ 1\end{array}\right)$. This state is an eigenstate of the operator $\sigma_{z}$ with eigenvalue -1 . If measuring the observable $\sigma_{z}$ in this state a certain value results: -1 ('no'). During the act of measurement the state $u$ is not changed since it is an eigenstate of the observable under discussion. Let us consider next a second measurement performed in state $u$, this time measuring the observable $\sigma_{x}$. Since the two eigenvectors of $\sigma_{x}$ are distinct from the state $u$ of the system, we get two different answers this time: +1 ('yes') and -1 ('no'). Both answers are uncertain; their probability is $1 / 2$ in both cases (reflecting maximal uncertainty). The state after the measurement of $\sigma_{x}$ is different from the state before: it represents a mixture of the two eigenstates of $\sigma_{x}$, namely $x^{-}$and $x^{+}$, both weighted with the same probability $1 / 2$.

The examples can be used to illustrate an important issue of quantum mechanics: preceding measurements can influence the actual state of the system and the results of the actual measurement when the operators representing the observables do not commute $\left(\sigma_{x} \sigma_{z} \neq \sigma_{z} \sigma_{x}\right)$. When first measuring $\sigma_{z}$ in the state $u$, we get a definite outcome and the state of the system does not change. When the observable $\sigma_{x}$ is measured before measuring $\sigma_{z}$, we get a uncertain result for the measurement of $\sigma_{z}$ since the first measurement has destroyed the state $u$ and transformed it into a mixed state. Section 6 will illustrate similar order phenomena in the domain of natural language semantics, especially in the context of attitude questions investigated in survey research and personality psychology.

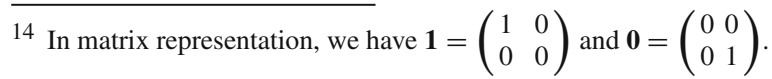


It is a simple exercise to prove that each Hermitian operator of the real vector space $\mathbf{R}^{2}$ can be written as a linear combination of $\sigma_{x}, \sigma_{z}$, and the identity matrix $\mathbf{I}$. In case of the complex vector space $\mathbf{C}^{2}$, we have to consider a third operator represented by the matrix $\sigma_{y}$ :

$$
\sigma_{y}=\left(\begin{array}{ll}
0 & i \\
-i & 0
\end{array}\right)
$$

The operator $\sigma y$ has the following two (normalized) eigenvectors: $y^{+}=\frac{1}{\sqrt{2}}\left(\begin{array}{l}1 \\ -i\end{array}\right)$ with eigenvalue +1 and $y^{-}=\frac{1}{\sqrt{2}}\left(\begin{array}{l}1 \\ i\end{array}\right)$ with eigenvalue -1 . The three operators $\sigma_{x}, \sigma_{y}$, and $\sigma_{z}$ are called the Pauli matrices (after the Austrian physicist Wolfgang Pauli). These operators are usually applied describing the spin of a spin-1/2 particles (such as electrons, neutrons and protons). Alternatively, they can also be used to describe the polarization of photons. The eigenstates of $\sigma_{x}$ and $\sigma_{z}$ describe states of linearly polarized photons whereas the eigenstates of $\sigma_{y}$ refer to states of circularly polarized photons (where the direction of polarization is not fixed but circulating around the axis of wave travelling).

\subsection{Qubits}

In classical information theory the states of a system are described in terms of two discrete units, say $\{0,1\}$. A decision between these two states requires one bit of information if the states have the same probability. In quantum computing, the qubit is considered as the elementary unit of information-deciding on a vector in a twodimensional complex vector space. Figure 7 depicts the situation. The left-hand side represents an (arbitrary) unit vector $u$ in terms of an azimuthal angle $\theta$ in case of real vector spaces. In the complex case, an additional parameter is required describing the phase shift between the two components of the state vector. This parameter is called the phase angle $\Delta$.

As an exercise, consider the state $u$ as depicted in Fig. 7. What is the probability for the +1 ('yes') answer when measuring the observable $\sigma_{z}$ ? Solution: the probability is $\cos ^{2}(\theta / 2)$ for real and complex vector spaces. The probability for the +1 ('yes') answer, when measuring the observable $\sigma_{x}$ (measuring the $x$-direction), is $1 / 2(1+\sin (\theta))$ for real vector spaces and $1 / 2(1+\sin (\theta) \cdot \cos (\Delta))$ for complex vector spaces. ${ }^{15}$ Hence, in the complex case, the phase factor shrinks the probability.

\footnotetext{
15 There are two equivalent ways of calculating the result. First, we can use the projector for the 'yes'answer, i.e. the matrix $\mathbf{1}=\left(\begin{array}{ll}1 & 0 \\ 0 & 0\end{array}\right)$, and multiply this matrix with the vector $u$ presented in Fig. 7. Next we calculate the square of the length of the resulting vector. The second possibility is to calculate the scalar product between $u$ and $z^{+}=\left(\begin{array}{l}1 \\ 0\end{array}\right)$ and calculate the square of the resulting amount. The details of these calculations are left for the reader.
} 


\section{Real Vector Space}

$$
u=\cos \theta / 2 \cdot\left(\begin{array}{l}
1 \\
0
\end{array}\right)+\sin \theta / 2 \cdot\left(\begin{array}{l}
0 \\
1
\end{array}\right)
$$

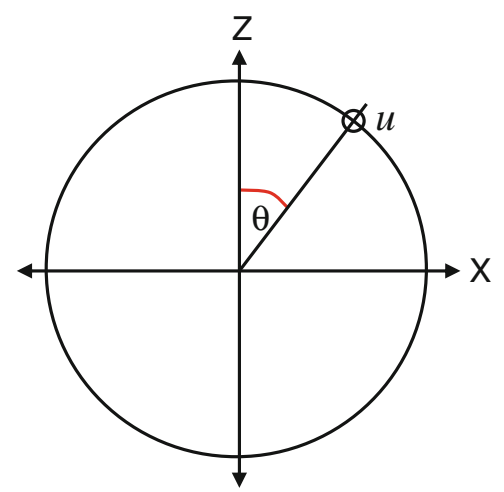

(a)
Complex Vector Space

$$
u=\cos \theta / 2 \cdot e^{-i \frac{\Delta}{2}} \cdot\left(\begin{array}{l}
1 \\
0
\end{array}\right)+\sin \theta / 2 \cdot e^{i \frac{\Delta}{2}} \cdot\left(\begin{array}{l}
0 \\
1
\end{array}\right)
$$

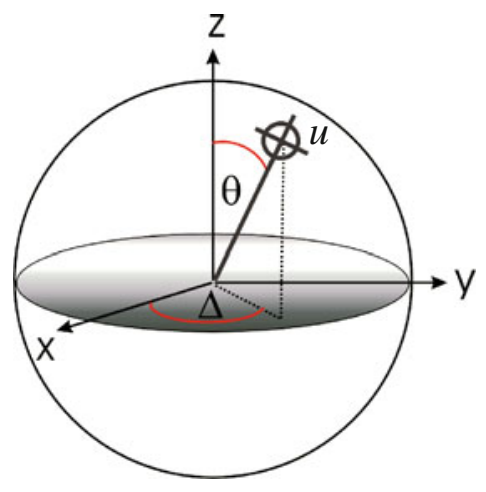

(b)

Fig. 7 a Realizing a qubit by selecting a state in a two-dimensional real vector space; $\mathbf{b}$ realizing a qubit by selecting a state in a two-dimensional complex vector space (Hilbert space). The latter figure is also called Bloch sphere

\subsection{The Application Function}

Consider the spectral decomposition defined in formula (16). For the following it is useful to have a function that selects exactly the part of the operator with a particular eigenvalue-let us call this function application. It is defined as follows:

(20) @ $\left(\mathbf{a}, \lambda_{\mathrm{i}}\right)=\mathbf{a}_{\mathrm{i}}$, where $\mathbf{a}_{\mathrm{i}}$ is the corresponding projection operator in the spectral decomposition (16)

The application device @ will be a useful instrument for formalizing the idea of a possible (full) answer to a question in Sects. 4 and 5.

\subsection{Quaternions}

A useful extension of the present framework is the introduction of quaternions. Quaternions are a generalization of complex numbers. The standard definition regards every quaternion as a unique and real linear combination of the basis quaternions $1, \mathrm{i}$, $\mathrm{j}$, and $\mathrm{k}: x=x_{0}+x_{1} \mathrm{i}+x_{2} \mathrm{j}+x_{3} \mathrm{k}$, with the set of equations $\mathrm{i}^{2}=\mathrm{j}^{2}=\mathrm{k}^{2}=\mathrm{ijk}=-1$. In principle, quaternions can be used like (complex) numbers. However, there is one important difference: the order of two quaternions can matter when multiplying them. In particular, we will use the quaternions $y$ and $n$ to represent the answers 'yes' and 'no', respectively (instead of numbers $+1,-1$ ). The point becomes visible in Sect. 4 
when we are concerned with the conjunction of questions ('do you like beer and do you like wine'). In such cases we need the possibility for representing composed answers (such as 'yes' to the first part and 'no' to the second part of the question). The mathematical framework from quantum physics has only numbers available to represent answers. This is not always very practical for applications of natural language semantics. As a compromise, I suggest the use of quaternions, which allows to distinguish between composed answers such as $y \cdot n$ and $n \cdot y$. Quaternions make sure that these composed answers are different since the commutative law $y \cdot n=n \cdot y$ is disobeyed.

\subsection{Orthomodularity and Inference}

Generally speaking, an orthomodular lattice (Rédei 2009) is the structure underlying propositions in a physical theory, which is based on a system of projection operators. A specific class of orthomodular lattices is the class of Hilbert lattices. A Hilbert lattice with lattice operations $\cap, \cup, \perp$ is the set of all projections of a complex, possibly infinitely dimensional Hilbert space $\mathscr{H}$. Note that the lattice operations $\cap, \cup$, and $\perp$ operate on projection operators. They correspond to the set-theoretical intersection, sum and orthocomplement operating on subspaces of $\mathscr{H} .{ }^{16}$ An orthomodular lattice is similar to a Boolean algebra but more general. Whereas a Boolean algebra satisfies distributivity (with regard to union and intersection), an ortho-modular lattice does not necessarily do so; it satisfies the axiom of orthomodularity, instead. ${ }^{17}$

The notion of inference can be defined in the following way, where $\mathbf{a}$ and $\mathbf{b}$ are projection operators:

$$
\mathbf{a} \models \mathbf{b} \text { iff } \mathbf{a b}=\mathbf{a}
$$

It is exactly the relation $\models$ which defines the lattice-theoretic properties of the orthomodular lattice.

For the following it is important to take the 'classical case' into account, where all the considered projection operators commute, i.e. are order-independent $(\mathbf{a b}=\mathbf{b a})$. In this case we have the following facts:

$$
\begin{array}{ll}
\text { a. } & \mathbf{a} \cap \mathbf{b}=\mathbf{a b} \\
\text { b. } & \mathbf{a} \cup \mathbf{b}=\mathbf{a}+\mathbf{b}-\mathbf{a b} \\
\text { c. } & \mathbf{a} \perp=\mathbf{I}-\mathbf{a} \text { (where } \mathbf{I} \text { is the identity operator introduced earlier) }
\end{array}
$$

\footnotetext{
16 More precisely, we can express this by introducing the range of a projector: $\|\mathbf{a}\|=\{u \in H: \mathbf{a} u=u\}$. Then the lattice operations on the space of projection operators are defined by the corresponding operations on vector (sub)spaces: $\|\mathbf{a} \cap \mathbf{b}\|=\|\mathbf{a}\| \cap\|\mathbf{b}\|,\|\mathbf{a} \cup \mathbf{b}\|=\|\mathbf{a}\|+\|\mathbf{b}\|$, and $\left\|\mathbf{a}^{\perp}\right\|=\|\mathbf{a}\|^{\perp}$.

17 I do not list the relevant axioms underlying an orthomodular lattice here. For a detailed exposition, the interested reader is referred to Rédei (2009). It should be noted, however, that the Hilbert lattice is not only non-distributive but it is also non-modular if the dimension of the Hilbert space is infinite. If the dimension is finite it is modular. The concept of an orthoalgebra is closely related to orthomodularity (Rédei 2009). Any orthomodular lattice determines an orthoalgebra. However, not every orthoalgebra is an orthomodular lattice. In this paper, we use the term 'orthoalgebraic approach' as a cover term for studying the algebraic properties of all projections of (finite and infinite) dimensional Hilbert spaces.

Another interesting point relates to the fact that distributivity is not the only property that distinguishes Boolean algebra from an orthomodular lattice. The existence of a cloning (or copying) operation is another property. It has been proven that an orthoalgebra admits cloning operation if and only if it is a Boolean algebra (Miyadera and Imai 2009). That is, only classical theory admits the cloning of states.
} 
In the last subsection, I introduced the two commuting projection operators $\mathbf{0}$ and $\mathbf{1}$, which realize the projections of the two base states $z^{+}$and $z^{-}$. Further, we used the notation I for the identity operator and $\varnothing$ for the zero-operator. All the operators $\mathbf{0 , 1} \mathbf{1}$ I and $\varnothing$ commute with each other and they can be seen as realizing a classical bit-system (as a subpart of the more general qubit-system).

\subsection{Tensor Product}

In quantum theory, complex systems are built by using the tensor product. Assume we have an orthonormal base $\mathbf{S}=\left(u_{1}, u_{2}, \ldots, u_{n}\right)$ of our Hilbert space $\mathscr{H}$ of dimension $\mathrm{n}$. By taking the tensor product, two vectors $u=\sum_{\mathrm{i}} x_{\mathrm{i}} \cdot u_{\mathrm{i}}$ and $v=\sum_{j} y_{j} \cdot u_{j}$ can be combined to form a joint state:

(23) $u \otimes v=\sum_{i j} x_{\mathrm{i}} y_{j} \cdot u_{\mathrm{i}} \otimes u_{j}$.

It is stipulated that the vectors $u_{\mathrm{i}} \otimes u_{j}$ form an orthonormal base of an $n^{2}$-dimensional Hilbert space $\mathscr{H}^{2}$. The notion of tensor product can straightforwardly be extended to linear operators. We will write $\mathbf{a} \otimes \mathbf{b}$ for the tensor product of the two operators $\mathbf{a}$ and b. It can be defined by stating the following condition:

(24) $\mathbf{a} \otimes \mathbf{b}(u \otimes v)=\mathbf{a} u \otimes \mathbf{b} u$, for all vectors $u, v \in \mathscr{H}$.

If the context excludes misunderstandings, it is convenient to leave out the symbol ' $\otimes$ '. For example, we will write 011 instead of $\mathbf{0} \otimes \mathbf{1} \otimes \mathbf{1}$. In the classical case all projection operators that are built from linearly combining pure projections in a $2^{n}$-dimensional Hilbert space (such as adding elements of $\mathbf{0 0 0 , 0 0 1 , 0 1 0}, \ldots$ in case of a 3 qubits) commute with each other. Consequently, they realize a Boolean algebra.

\section{Orthoalgebraic Semantics}

In the last section, we have seen that each normal operator/observable a in a Hilbert space $\mathscr{H}$ can be decomposed in pairwise orthogonal subspaces (described by projection operators) which are decorated by the corresponding eigenvalues of $\mathbf{a}$. The sum of these subspaces gives the whole Hilbert space $\mathscr{H}$; hence we can speak of a decorated partition of $\mathscr{H}$. In this section, I will investigate the idea of decorated partitions further, and I will use this idea for developing a new version of question semantics.

We can exclude the kernel (null space) of an observable (i.e. the vector space corresponding to eigenvectors with eigenvalue zero) from the partition since it does not contribute to the sum of the spectral decomposition in (16). In this way, an observable a can effectively be represented by a decorated partition of the non-null part of the observable.

\subsection{The Query Language QL* and Its Semantics}

We start from a standard query language $Q L^{*}$, which is built from a set of propositional variables $p, q, r, \ldots$, with the help of negation $\neg \varphi$, conjunction $\varphi \wedge \psi$, disjunction $\varphi \vee \psi$, declarative $! \varphi$ and question ? $\varphi$. The semantics of this language is defined relatively to a Hilbert space $\mathscr{H}$ (and the orthomodular lattice defined on it). For defining 
the semantics, we assume an assignment function $\pi$ which assigns projection operators in $\mathscr{H}$ to the propositional variables. Then the semantic values for the formulas of $Q L^{*}$ are defined as follows:

$$
\begin{array}{ll}
\text { a. } & \ll p \gg=\pi(p) \\
\text { b. } & \ll \neg \varphi \gg=\Sigma_{\mathrm{i}} \lambda_{\mathrm{i}} \mathbf{a}_{\mathrm{i}}^{\perp} \text { where } \ll \varphi \gg=\Sigma_{\mathrm{i}} \lambda_{\mathrm{i}} \cdot \mathbf{a}_{\mathrm{i}} \text { (the spectral decomposition } \\
& \text { of }\langle\varphi \gg) \\
\text { c. } & \ll \varphi \wedge \psi \gg=\ll \psi \gg \ll \varphi \gg \\
\text { d. } & \ll ! \varphi \gg=(\text { null } \ll \varphi \gg)^{\perp} \\
\text { e. } & \ll ? \varphi \gg=y \cdot \ll \varphi \gg+n \cdot \ll \neg \varphi \gg(\text { ' } y \text { ', ' } n \text { ' are quaternions for 'yes', } \\
& \text { 'no') }
\end{array}
$$

Notice that the semantic value of $\neg \varphi$ is a projection operator again if $\varphi$ is a projection operator (25b). In this case, we have $\ll \neg \varphi \gg=\mathbf{I}-\ll \varphi \gg$. If $\varphi$ is a yes/no-question, then $\neg \varphi$ turns the yes-answer into the no-answer and the no-answer into the yes-answer.

The semantic value of the conjunction is the composition of the corresponding operators, as defined in (25c). Using the result of (22a), this conforms to the intersection operation in case the order of the operators does not matter (commutativity). The general case of non-commuting operators will be considered in Sect. 6. Note that the composition of two Hermitian operators is Hermitian again only if both operators commute.

The semantic value of declarative $! \varphi$ as defined by (25d) is a projection operator that projects into the orthocomplement of the kernel of $\ll \varphi \gg .{ }^{18}$ If $\varphi$ expresses a proposition, then the semantic value of $! \varphi$ is the same as the semantic value of $\varphi$ itself, namely a projection operator that projects into the corresponding subspace of $\mathscr{H}$. If $\varphi$ expresses a yes/no-question, then the semantic value of ! $\varphi$ is a projection that projects into the whole Hilbert space $\mathscr{H}$. This tells us that the question raises an issue, but does not provide any information.

Equation (25e) gives the definition of the question operator (yes/no-questions). If $\varphi$ expresses a proposition, then the question operator transforms this proposition into the corresponding yes/no-question.

Two further definitions are required:

$$
\begin{aligned}
& \text { a. } \quad \varphi \vee \psi=\neg(\neg \varphi \wedge \neg \psi) \\
& \text { b. } \varphi \rightarrow \psi=\varphi^{\perp} \vee(\varphi \wedge \psi)
\end{aligned}
$$

The first definition (26a) looks very classical. In fact, it corresponds to the classical Boolean operation of disjunction if $\varphi$ and $\psi$ are declaratives. The implication defined in (26b) is the Sasaki implication well-known from quantum logic (cf. Dunn and Hardegree 2001), the algebraic properties of which were investigated by Amira et al. (1998), inter alia. Interestingly, these definitions also apply if $\varphi$ and/or $\psi$ are questions. This leads to surprising results, which will be discussed after the introduction of some basic semantic concepts.

\footnotetext{
18 Note that we write 'null a' for the projection operator corresponding to the kernel of a, denoted by null a. The first expression refers to an operator; the latter expression refers to a vector space (the null space). Both notations are closely related, of course.
} 


\subsection{Truth and Probability}

A declarative formula $\varphi$ is semantically represented by a projection operator $\langle\varphi$ 》. A declarative $\varphi$ is considered 'true' in a situation $u$ iff $u$ is an eigenvector of $\ll \varphi \gg$ with eigenvalues 1 ; formally:

$$
u \vdash \varphi \quad \text { iff } \ll \varphi \gg u=u
$$

In Sect. 3.4 (Fig. 5) we have introduced the Born rule which says that the probability of getting the value 1 ('yes') when measuring the projection operator a in state $u$ is the square of the length of the vector $\mathbf{a} u$. In other words, $\|\mathbf{a} u\|^{2}$ is the probability that $u$ collapses in the eigenspace of a with eigenvalue 1 when measuring it. If $\mathbf{a}$ is the semantic value of a declarative $\varphi$ (i.e. $\mathbf{a}=\ll \varphi \gg$ ), then the squared length of $\ll \varphi \gg u$ is the probability that $\varphi$ is true for state $u$. In the following, I will use a special notation for expressing the Born rule:

$$
[[\varphi]]_{u}=\|\ll \varphi \gg u\|^{2}
$$

It is easy to prove that for declaratives $\varphi$ and states $u \neq 0, u \vdash \varphi$ iff $[[\varphi]]_{u}=1$ and $u \mid-\neg \varphi$ iff $[[\varphi]]_{u}=0$. As an exercise the interested reader can check that $[[\neg \varphi]]_{u}=1-[[\varphi]]_{u}$ and $[[\varphi \vee \psi]]_{u}=[[\varphi]]_{u}+[[\psi]]_{u}-[[\varphi \wedge \psi]]_{u}$, where $\varphi$ and $\psi$ are declaratives. The latter rule holds for commuting operators only: $\langle\varphi \gg\langle\psi \gg=$ $\ll \psi \gg \ll \varphi \gg$.

At this place a remark about sequences of declaratives and conjunctions of declaratives is in order. Standard conjunction in quantum logic (e.g. Rédei 2009) is always a symmetrical operation, i.e. the order of the two propositions that are conjoined does not matter. Sequences, in contrast, can be order-dependent in the general case. Hence, exactly in the 'classical' case of commuting observables, the notion of conjunction and the notion of sequence coincide.

One could think that a proper definition of a sequence of two observables is their composition. Unfortunately, this is not generally correct since the composition of two Hermitian operators is only Hermitian if the operators commute. There is a trick that is used in quantum theory to overcome this problem. The trick is to repeat the first operator at the end of the sequence. In this way, the system is forced into the eigenstates of the first operator. Using this trick, we can define the semantic value of the sequence $(\varphi ; \psi)$ as follows:

(29) $\langle\varphi ; \psi \gg=\langle\varphi \gg \ll \psi \gg \ll \varphi \gg$

Consequently, the probability of the proposition expressed by the sequence $(\varphi ; \psi)$ in state $u$ is given by the formula $[[\varphi ; \psi]]_{u}=\|\left\langle\varphi \gg\langle\psi\rangle \gg\left\langle\varphi \gg u \|^{2}\right.\right.$. Recently, Niestegge (2008) has shown that this method is more than just a trick. Following Lüders' transformation theory of measurement (Lüders 1951), his argument is that it makes complete sense to introduce a conditioned quantum probability by using sequences:

(30) $[[\psi / \varphi]]_{u}=[[\varphi ; \psi]]_{u} /[[\varphi]]_{u}$.

This formula resembles the classical definition of conditioned probability: $\mathrm{P}(\psi / \varphi)=$ $P(\varphi \wedge \psi) / P(\varphi)$. For more arguments that this method can usefully be applied for defining the semantics of sequences see the original literature and Blutner (2009). In Sect. 6, I will give an example of how to use these conditioned quantum probabilities. 


\subsection{Span of an Operator}

For comparing the orthoalgebraic approach with classical approaches to questions and answers, I will introduce the notion of span. The span of a normal operation a is defined as the relation between the eigenstates of $\mathbf{a}$ that have the same (non-zero) eigenvalues:

$$
\operatorname{span} \mathbf{a}=\{(u, v): \mathbf{a} u=\lambda u \text { and } \mathbf{a} v=\lambda v \text { for some } \lambda \neq 0\}
$$

Obviously, the span of a normal operator is an equivalence relation. Now we consider the query language $Q L^{*}$ with the interpretation as given in (25). Take an expression $\varphi$ interpreted as $\langle\varphi\rangle$. Instead of writing span $\langle\varphi \gg$ for the span of the corresponding operator we will simply write $\operatorname{span} \varphi$. For expressions that are interpreted by project-ion operators (declaratives) the span gives exactly the information that is provided by the truth conditions. Hence, we have the following facts for declaratives $\varphi$ :

$$
\begin{aligned}
& \text { a. } \quad(u, v) \in \operatorname{span} \varphi \text { iff } u \vdash \varphi \text { and } v \vdash \varphi \\
& \text { b. } u \vdash \varphi \text { iff }(u, u) \in \operatorname{span} \varphi .
\end{aligned}
$$

I will now consider the classical case in which all operators commute with each other. If $\varphi$ and $\psi$ are declaratives, then the spans of $\neg \varphi, ? \varphi$, and $\varphi \rightarrow \psi$ can be calculated as follows:

$$
\begin{array}{ll}
\text { a. } & \operatorname{span} \neg \varphi=\{(u, v):(u, u) \notin \operatorname{span} \varphi \&(v, v) \notin \operatorname{span} \varphi\} \\
\text { b. } & \operatorname{span} ? \varphi=\{(u, v):(u, u) \in \operatorname{span} \varphi \Leftrightarrow(v, v) \in \operatorname{span} \varphi\} \\
\text { c. } & \operatorname{span} \varphi \rightarrow ? \psi=\{(u, v): \operatorname{if}(u, v) \in \operatorname{span} ? \varphi \&(u, v) \in \operatorname{span} \varphi \operatorname{then}(u, v) \in \\
& \operatorname{span} ? \psi\}
\end{array}
$$

Interestingly, these clauses conform exactly to the question semantics as proposed by $\mathrm{JH}$. We can see this by applying the expressions in the update formulation of the $\mathrm{JH}$ approach (6) to the information state $\sigma=W^{2}$ (expressing a tautology). The clause (33a) corresponds to clause (6b), (33b) corresponds to (6d), and (33c) corresponds to (6e).

For deriving a more complete correspondence between the JH approach and the orthoalgebraic approach let us introduce the flat fragment of $Q L$. It consists of all declarative statement of $Q L$, questions ? $\varphi$ and conditional questions $\varphi \Rightarrow \psi$ (where $\varphi$ and $\psi$ are declaratives). Further, we allow the conjunction of both (conditional) questions and declaratives. The flat fragment of $Q L$ can be translated into a corresponding fragment of $Q L^{*}$ by assuming the following translation rules: $p^{\prime}=p$ (for atomic symbols $p$ of $Q L) ;(\neg \varphi)^{\prime}=\neg \varphi^{\prime} ;(\varphi \wedge \psi)^{\prime}=\varphi^{\prime} \wedge \psi^{\prime} ;(? \varphi)^{\prime}=? \varphi^{\prime} ;(\varphi \Rightarrow \psi)^{\prime}=\varphi^{\prime} \rightarrow \psi^{\prime}$. I will assume that we can express any information state $\sigma$ by a sequence $\Sigma$ of formulas of $Q L^{*}: \sigma=\operatorname{span} \Sigma$.

The following fact can be proven for the flat fragment of $Q L$ :

$$
\sigma \ll \xi \gg=\operatorname{span}\left(\Sigma ; \xi^{\prime}\right) \text { for any expression } \xi \text { of the flat fragment of } Q L \text {. }
$$

Note that this scheme is not valid for all expressions of $Q L$, for example it does not hold for expressions such as ?? $p$. Since such expressions are difficult to interpret anyway, it is not a real problem to exclude them and to concentrate on the flat fragment. 
Let us prove fact (34) now. First, consider the case where $\xi$ is an atomic formula, say $p$. Then we can assume that the set of possible worlds that is assigned to the atomic symbol via the interpretation function $\omega$ defines the projection operator $\pi(p)$ needed for interpreting the language $Q L^{*}$. We simply have to assume that $\pi(p)$ is a projection operator that projects into the vector space spanned by $\omega(p)$. Formally, this can be expressed in two different ways:

(35) a. $\quad \pi(p)=\sum_{u} \in \omega_{(p)} \mathbf{a}_{u}$, where $\mathbf{a}_{u}$ is a projection operator projecting each vector of into the one-dimensional subspace hulled by $u$; see (15).

b. $\omega(p)=\operatorname{null}(\pi(p)-\mathbf{I})$

To cite an example, consider the fragment of $Q L$ discussed in Sect. 2. It consists of two atomic symbols $p$ and $q$ and the assignment function is $\omega(p)=\{10,11\}$ and $\omega(q)=\{01,11\}$. Conforming to the treatment in Sect. 4.1, the corresponding projection operators are $\pi(p)=\mathbf{1 0}+\mathbf{1 1}$ and $\pi(q)=\mathbf{0 1}+\mathbf{1 1}$. Using the notion of span introduced in (31), we can state the following fact:

(36) $\operatorname{span} p=\{(u, v): u \in \omega(p)$ and $v \in \omega(p)\}$, for any atomic symbol $p$ of $Q L$.

Next, it is easy to show that in case of commuting declaratives we observe that

$$
\operatorname{span}\left(\varphi^{\prime} ; \psi^{\prime}\right)=\operatorname{span} \varphi^{\prime} \cap \operatorname{span} \psi^{\prime}
$$

As a consequence, we get

$$
\begin{aligned}
\operatorname{span}(\Sigma ; p) & =\operatorname{span} \Sigma \cap \operatorname{span} p=\sigma \cap \operatorname{span} p \\
& =\sigma \cap\{(u, v): u \in \omega(p) \text { and } v \in \omega(p)\} \\
& =\sigma \ll p \gg \quad(6 a)
\end{aligned}
$$

This concludes the proof that (34) is valid in case of $\xi=p$.

Second, we consider an expression $\neg \xi$, where $\xi$ is a propositional formula. It can be shown now that $\sigma \ll \neg \xi \gg=\operatorname{span}(\Sigma ; \neg \xi)$ :

$$
\begin{aligned}
\operatorname{span}(\Sigma ; \neg \xi) & =\operatorname{span} \Sigma \cap \operatorname{span} \neg \xi=\sigma \cap \operatorname{span} \neg \xi \\
& =\sigma \cap\{(u, v):(u, u) \notin \operatorname{span} \xi \text { and }(u, u) \notin \operatorname{span} \xi\} \\
& =\sigma \ll \neg \xi \gg(6 \mathrm{~b})
\end{aligned}
$$

Third, for plain questions ? $\xi$ it can be shown that $\sigma \ll ? \xi \gg=\operatorname{span}(\Sigma ; ? \xi)$. The proof rests on a simple generalization of (33b) which introduces an initial information state $\sigma=\operatorname{span} \Sigma$. The generalization is:

$$
\text { b'. }^{\prime} \operatorname{span}(\Sigma ; ? \varphi)=\{(u, v):(u, u) \in \operatorname{span}(\Sigma ; \varphi) \Leftrightarrow(v, v) \in \operatorname{span}(\Sigma ; \varphi)\} .
$$

In a similar way, the equivalence $\sigma \ll \varphi \Rightarrow$ ? $\psi \gg=\operatorname{span}(\Sigma ; \varphi \rightarrow$ ? $\psi)$ can be shown for proposit-ional formulas $\varphi$ and $\psi$. Finally, for declaratives and plain questions $\varphi$ and $\psi$ it can be shown that $\sigma \ll \varphi \wedge \psi \gg=\operatorname{span}(\Sigma ; \varphi \wedge \psi)$. 
Fig. 8 Picture of meaning ? $p$ in orthoalgebraic semantics
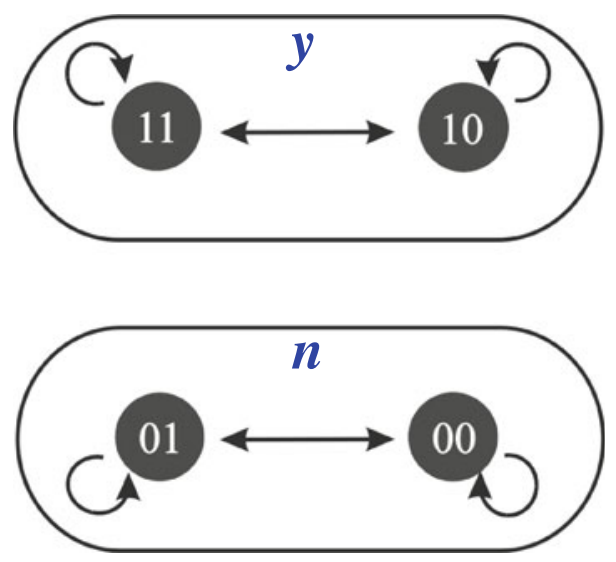

What we can conclude from these considerations is that essential parts of the $\mathrm{JH}$ approach can be extracted from the operator formalism introduced in this section. In particular, we can derive the basic clauses (6) of the JH approach by considering the span of the corresponding operators in the orthoalgebraic approach.

Despite the existence of an extraction mechanism, it should be stressed that the present operator approach and the JH approach are not equivalent. The operator approach is more powerful than the $\mathrm{JH}$ approach for two reasons. First, it is able to handle question order effects (non-commuting operators). This will be discussed in Sect. 6. Second, the operator formalism is more structured than the JH system even in the case of commuting operators. The point is that the orthoalgebraic approach considers decorated partitions instead of standard partitions. In order to see the important differences let us consider some simple examples in the next subsection. In Sect. 5 we will continue the discussion by considering some generalizations.

\subsection{Examples with Commuting Operators}

In Fig. 2 (Sect. 2) the meaning of ?p in the JH question semantics was pictured. We see two equivalence classes which partition the space $W$ of possible worlds. Figure 8 depicts the meaning of $? p$ in orthoalgebraic semantics. We see the same equivalence classes, but now the two blocks are decorated by the quaternions $y$ and $n$, respectively, corresponding to the two possible answers yes and no.

The meaning of the conditional interrogative $p \Rightarrow ? q$ was pictured in Fig. 3 for the JH semantics. Figure 9 shows the meaning of the related expression in the orthoalgebraic framework.

The derivation of the relevant partition is as follows:

$$
\begin{aligned}
\ll p \rightarrow ? q \gg & =\neg(\mathbf{1 0}+\mathbf{1 1})+(\mathbf{1 0}+\mathbf{1 1})(y(\mathbf{0 1}+\mathbf{1 1})+n(\mathbf{1 0}+\mathbf{0 0})) \\
& =(\mathbf{0 0}+\mathbf{0 1})+(y \mathbf{1 1}+n \mathbf{1 0})
\end{aligned}
$$


Fig. 9 Picture of meaning $\mathrm{p} \rightarrow$ ?q in orthoalgebraic semantics

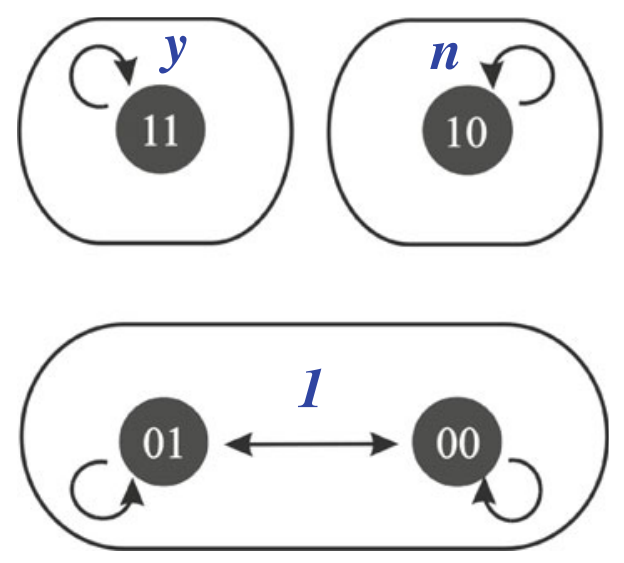

Fig. 10 Picture of meaning $? p \wedge ? q$ in orthoalgebraic semantics
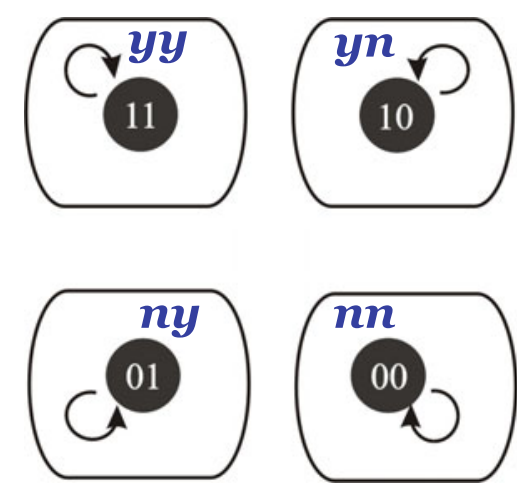

In both cases, the partition of the logical space consists of three blocks, corresponding to the propositions expressed by $p \wedge q, p \wedge \neg q$ and $\neg p$. In the latter case these propositions are decorated: $p \wedge q$ by $y, p \wedge \neg q$ by $n$, and $\neg p$ by 1 . As before, we can take the first two decorations to indicate the traditional answer types yes and no; and we can take the decoration 1 to indicate the condition for a supposition failure.

The following example shows the composition of two questions ? $p$ and ?q forming the new question $? p \wedge ? q$. Figure 10 depicts the corresponding meaning using the composed decorations $y y, y n, n y$, and $n n$.

Obviously, the parts of the complex quaternions refer to the corresponding subquestions, e.g. in the world 10 the question ? $p$ is answered by $y$ and the question ? $q$ is answered by $n$. At this point we also see the motivation for using quaternions instead of simple numbers for indicating composed answers. If we had used +1 for 'yes' and -1 for 'no', we could not distinguish a 'yes'-'no' answer form a 'no'-'yes' answer since we would get -1 in both cases. Similarly we could not distinguish a 'yes'-'yes' answer from a 'no'-'no' answer ( +1 in both cases). 


\subsection{Congruent Answers}

An important empirical problem, which any theory of questions and answer has to solve, relates to the proper characterization of congruent answers (e.g. Groenendijk and Stokhof 1997; Krifka 2001). In the simplest case of constituent questions, a congruent question is just an answer that fills in a constituent for the wh-expression in the question. And a congruent full answer is just the question meaning applied to the term-answer.

More formally, this idea can be expressed in the following way by the use of the application device @ defined in (20):

(37) $\varphi$ is a congruent full answer to a question $\psi$ iff $@(\ll \psi \gg, t)=\langle\varphi \gg$ for some element $t$ of the spectrum of $\ll \psi \gg$.

A simple example is in order. Clearly, the assertion $p$ is a congruent answer to ? $p$. This derives from the observation that $@(\langle ? p \gg, y)=\langle p \gg$. Similarly, $\neg p$ is a proper answer to ?p since @ $(\ll ? p \gg, n)=\ll \neg p \gg$.

Now consider the following utterance of a question (38a) made by a competent speaker. Congruent answers are the conditional answers presented in (38b,c). Intuitively, composed answers such as in $(38 \mathrm{~d}, \mathrm{e})$ do not count as congruent answers and are not very appropriate.

(38) a. If Mary reads this book, will she recommend it to Peter?

b. Yes. If Mary reads this book, she will recommend it to Peter.

c. No. If Mary reads this book, she will not recommend it to Peter.

d. *Yes. Mary reads this book, and she will recommend it to Peter.

e. *No. Mary reads this book, and she will not recommend it to Peter.

Interestingly, the $\mathrm{JH}$ approach does not predict the proper conditional answers but the conjoined answers. How to handle this problem in orthoalgebraic semantics? Does the definition given in (37) generalize to the idea of congruent answers in case of conditional questions?

Unfortunately, this does not work in the case of conditional questions. However, a simple adjustment is possible and provides the proper generalization. The proposal is to change the definition by taking the proposition with the decoration1into account. As mentioned above, this proposition expresses the condition for a supposition failure (see Fig. 9 for an example).

(39) $\varphi$ is a congruent full answer to a question $\psi$ iff $@(\ll \psi \gg, t)+@(\ll \psi \gg, 1)=$ $\ll \varphi \gg$ for some element $t$ of the spectrum of $\langle\psi \gg$.

A consequence of this definition is that congruency is possible only with conditional answers for conditional questions. For instance, $\ll p \rightarrow q \gg$ comes out as a congruent full answer to $\ll p \rightarrow ? q \gg$. We can derive this fact from the equivalences $\ll p \rightarrow$ $? q\rangle=\left\langle\langle p\rangle^{\perp}+\left\langle\langle p\rangle\left\langle\langle q q\rangle=1\left\langle\langle p\rangle^{\perp}+y\left\langle\langle p\rangle\left\langle\langle q\rangle+n\langle\langle p\rangle q\rangle^{\perp}\right.\right.\right.\right.\right.\right.$. The application @ $(\ll p \rightarrow ? q \gg, y)$ results in $\ll p\rangle^{\perp}$ and the application $@(\ll p \rightarrow$ $? q \gg, 1)$ results in $\left\langle p p \gg\left\langle\langle q\rangle\right.\right.$. Consequently, the sum gives $\langle p\rangle^{\perp}+\langle p \gg\langle\langle q\rangle$, which is nothing else than $\ll p \rightarrow q \gg$. Hence, according to definition (33), $\ll p \rightarrow$ $q \gg$ comes out as a congruent answer to $\ll p \rightarrow ? q \gg$. 
Similarly, $\ll p \rightarrow \neg q \gg$ can be shown to be a congruent full answer to $\ll p \rightarrow ? q \gg$ :

$$
\begin{aligned}
@(\ll p \rightarrow ? q \gg, n)+@(\ll p \rightarrow ? q \gg, 1) & \left.=\ll p\rangle^{\perp}+\ll p \gg \ll q\right\rangle^{\perp} \\
& =\ll p \rightarrow \neg q \gg .
\end{aligned}
$$

Further, since the two full answers given before are the only congruent answers to the question $\ll p \rightarrow ? q \gg, \ll p \wedge q \gg$ cannot be a proper answer to $\ll p \rightarrow ? q \gg$.

\subsection{Preliminary Conclusions}

Concluding this section, I claim that the present approach explains why informationally equivalent questions like "Is the door open?" and "Is the door closed?" have different meanings. Further it overcomes the conceptual imperfection of the JH approach: only one definition of the conditional is required in order to capture both the usual material implication of declaratives and the interrogative conditional connecting a declarative antecedent with a question. It also overcomes the main empirical problems of the $\mathrm{JH}$ approach due to the uniformity of the classical partition semantics which gives all partitions the same status. In the case of the interrogative conditional, it indicates when an 'answer' counts as a species of presupposition failure. Moreover, it is simple to show that in orthoalgebraic semantics the equivalence between $p \rightarrow p$ ? and $p p$ is no longer valid. And it eliminates perhaps the biggest drawback of the JH approach: that it counter-intuitively predicts conjunctive answers for conditional questions. ${ }^{19}$

\section{Comparison with the Structured Meaning Approach}

In a seminal paper, Krifka (2001) argued for a structured meaning account of questions and answers (see also Krifka 2004). He demonstrated that the GS partition theory (and related approaches summarized as proposition set approaches by Krifka) runs into three problems:

It does not always predict the right focus structure in answers, it is unable to distinguish between polarity (yes/no) and a certain type of alternative questions, and it does not allow to formulate an important condition for a type of multiple constituent questions. (Krifka 2001, p. 287).

Further, Krifka made clear in the same paper that the structured meaning approach can handle all three problems. Without going into a detailed discussion here, I will illustrate only the close correspondence of the structured meaning account and the present decorated partition theory. Krifka summarizes the basic idea underlying the

\footnotetext{
19 There are other puzzles besides the discussed ones. One refers to unconditionals (Zaefferer 1991), exemplified by sentences such as "Whether you like it or not, your talk was simply boring" (Zaefferer 1991: p. 488). Several authors have suggested analyzing unconditionals by presuming questions in the antecedent of the conditional (Arita and Kaufmann 2008; Isaacs and Rawlins 2008; Rawlins 2008). We have to leave an analysis of unconditionals by using the present framework for another occasion.
} 
structured meaning approach as follows: "Question meanings are functions that, when applied to the meaning of the answer, yield a proposition." (Krifka 2001, p. 288).

When we use the application device proposed in the previous section (instead of the operation of functional application in a categorical language as proposed by Krifka), then we see immediately that our decorated partition semantics shares a basic trait with the structured meaning approach: question meanings can be applied to the meaning of (term-)answers yielding a proposition. Interestingly, the presented application device @ and the definition of congruent answers as given in (39) is also valid for conditional questions. $^{20}$

As far as I can see, the structured meaning approach has not been applied to conditional questions yet. It is an interesting task to extend this approach in order to include conditional questions. At the end of this section I will make an explicit proposal for that. This proposal simply implements the idea underlying definition (39) within the structured meaning approach.

In case of commuting operators, the orthoalgebraic semantics of Sect. 4 can be translated into a traditional format. If all operators commute, we have a common system of orthonormal eigenvectors. This system of eigenvectors can be identified with the set of possible worlds $W$. In the structured meaning approach, information states are no longer defined by equivalence relations describing partitions of $W$. Instead, they are defined by decorated partitions which can be represented by functions $f$ from $W$ onto some domain. The values of this function are the decorations of the relevant equivalence classes. Since we do not consider $w h$-questions in this paper but only (conditional) yes/no-questions, the relevant domain consists of the elements 0,1 , $y, n$.

Let us consider the query language $Q L$ from Sect. 2. The semantics provided in Sect. 2 formulated the information change potential $\ll \xi \gg$ of an expression $\xi$ of $Q L$ as a function from information states to information states. In Sect. 2, information states were considered as equivalence relations over the logical space, $\sigma \subseteq W^{2}$. Instead of equivalence relations, now we consider information states as functions $f$ from $W$ into the domain $\{0,1, y, n\}$. The following update clauses are stipulated where $\omega$ is a classical interpretation function (assigning subsets of $W$ to the atomic propositional symbols):

(40) a. $f \ll p \gg=\lambda u . f(u) \cdot \chi_{u}[\omega(p)]$, where $\chi_{u}(\mathrm{X})$ is the characteristic function of set $\mathrm{X} .^{21}$

\footnotetext{
${ }^{20}$ It should be mentioned that a major advantage of the structured meaning approach is that it can effortlessly deal with term answers ("who will organize this workshop?-Peter!"). In the present paper we discuss sentential answers only, which also can be treated by the structured meaning approach (e.g., Von Stechow 1991). It is an interesting exercise to extend the present approach including a proper treatment of term answers. Recent research on a compositional approach to distributional semantics using a compositional theory for grammatical types (e.g., Coecke et al. 2011) might bolster a sound treatment of term questions and term answers.

$21 \chi_{u}(X)=\operatorname{def} \begin{cases}1 & \text { if } u \in \mathrm{X} \\ 0 & \text { if } u \notin \mathrm{X}\end{cases}$

Note that the sign "." on the right hand site of equation (40a) is a real multiplication sign: e.g., $x ? 0=0$ and $x ? 1=x$. Hence, if we start with the constant function $f(u)=1$ (representing the 'empty' information state $W^{2}$ ), then updating $f$ with information $\ll p \gg$ yields the characteristic function of the proposition $\ll p \gg$, i.e. $\chi_{u}[\omega(p)]$.
} 
b. $\quad f \ll \neg \varphi \gg=\lambda u . \overline{f \ll \varphi \gg u}$ (with $\overline{0}=1, \overline{1}=0, \bar{y}=n, \bar{n}=y$ )

c. $f \ll \varphi \wedge \psi \gg=f \ll \varphi \gg \ll \psi \gg$

d. $f \ll ? \varphi \gg=\lambda u . \begin{cases}y, & \text { if } f \ll \varphi \gg u=1 \\ n, & \text { if } f \ll \varphi \gg u=0\end{cases}$

e. $f \ll \varphi \Rightarrow \psi \gg=\lambda u .\left\{\begin{array}{l}1, \quad \text { if } f \ll \varphi \gg u=0 \\ f \ll \varphi \wedge \psi \gg u, \text { elsewhere }\end{array}\right.$

In the classical case of commuting operators, the operator formalism from Sect. 4 and the present formulation agree. Let us introduce the notion of $d$-span (defining a decorated partition for linear operators). In close analogy to the notion of span defined in (31) we assume that all considered operators commute and we identify the common system of eigenvectors with the set of possible worlds $W$.

(41) d-span $\mathbf{a}=$ the function $f$ defined on $W$ such that $f(u)=$ the eigenvalue of $\mathbf{a}$ in state $u \in W$.

Let us assume that a given information state $f$ can be represented by a sequence of expressions $\Sigma$ of $Q L: f=\mathbf{d}$-span $\Sigma$. We can prove then the equivalence of the orthoalgebraic semantics with the present structured meaning approach as expressed by the update clauses in (40). More precisely, it can be proven that for any expression $\xi$ of the flat fragment of the query language $Q L$ the following proposition is valid:

(42) $f \ll \xi \gg=\mathbf{d}$-span $\ll \Sigma$; $\xi^{\prime} \gg$, where $\xi^{\prime}$ is the translation of $\xi$ into $Q L^{*}$

For the proof, we assume pairwise commuting operators. We get

$$
\begin{aligned}
& \text { d-span } \ll \Sigma ; \xi^{\prime} \gg u=\mathbf{d}-\operatorname{span} \ll \Sigma \gg u \cdot \mathbf{d}-\operatorname{span} \ll \xi^{\prime} \gg u=f(u) \cdot \mathbf{d}-\text { span } \\
& \ll \xi \gg^{\prime} u
\end{aligned}
$$

The equivalence (42) is easily shown for any atomic symbol $p$. Here we get

$$
\text { d-span } \ll \Sigma ; p \gg u=f(u) \cdot \mathbf{d}-\mathbf{s p a n} \ll p \gg u
$$

Further, it is $\ll p \gg=\pi(p)$ and for the operator $\pi(\mathrm{p})$ the eigenvalue of a state $u \in W$ is 1 if $u \in \omega(p)$ and 0 if $u \notin \omega(p)$. Hence, we get d-span $\ll p \gg(u)=\chi_{u}[\omega(p)]$. Consequently, we get

$$
\text { d-span } \ll \Sigma ; p \gg u=f(u) \cdot \chi_{u}[\omega(p)]
$$

Comparing this result with the information change expressed by (40a), we see the sameness. This verifies the equivalence (42) for $\xi=p$. It is left for the interested reader to complete the proof and the show the equivalence for the other clauses.

In Sect. 3.7, the application device @ for linear operators was defined. A similar device can be formulated for the structured meaning account with reference to information states $f$ :

$$
@(f, \lambda)=\{u: f(u)=\lambda\}
$$

The application functor @ takes an information state $f$ and a possible value $\lambda$ of the function $f$ and calculates the set of possible worlds that yield exactly the value $\lambda$ when $f$ is applied to them. Definition (39) of a congruent full answer can now be translated into the following form conforming to the structured meaning approach: 
(45) $\varphi$ is a congruent full answer to a question $\psi$ in information state $f$ iff $@(f \ll \psi \gg, \mathrm{t}) \cup @(f \ll \psi \gg, 1)=f \ll \varphi \gg$ for some element $\mathrm{t}$ of the set $\{0,1, y, n\}$.

For example, we can take $f$ to be the empty information state over $W=$ $\{10,11,01,11\}$ (i.e., $f(u)=1$ for all $u \in c W)$. Further, the interpretation function is $\omega(p)=\{10,11\}$ and $\omega(q)=\{01,11\}$. Then the applications $@(f \ll p \Rightarrow$ $? q \gg, y)=\{11\}$ and $@(f \ll p \Rightarrow ? \gg, 1)=\{00,01\}$ can be calculated by the use of (40f). Correspondingly, the union of these two propositions is $\{11,00,01\}$. This is identical to the proposition $f \ll p \rightarrow q \gg$. Hence, $p \rightarrow q$ is a congruent full answer to the question $p \Rightarrow ? q$ in the information state $f$. Obviously, the example shows that the translation of the operator formalism into the more standard account of structured meaning works properly.

Taking the present result into account, one could argue that a semanticist interested in query languages could ignore the orthoalgebraic formalism completely and develop his theories completely by using standard techniques of elementary set theory. Although this is true to some extent, it misses out the important issues of generality, uniformity and systematicity that are intrinsic to the orthoalgebraic account. These are advantages that cannot be ignored for theoretical reasons. In my opinion, there are three main arguments for continuing to use the operator framework. First, there is the methodological aspect of understanding how quantum theory in physics relates to question theories in formal semantics. Crossing boundaries seems to be useful for both disciplines. Second, I think that the orthoalgebraic formalism gives a very concise and elegant description of a theory of question and answers (assuming the reader is familiar with some basic elements of linear algebra). Section 4.2 has shown that this approach has the potential to handle situations of uncertainty-a very important aspect of question semantics. Third, this formalism straightforwardly generalizes to the non-commutative case. Hence, it can be used to model phenomena of opinion forming as discussed in survey research (Schuman and Presser 1981) and personality diagnostics (Blutner and Hochnadel 2010). The following section is devoted to the issue of non-commutativity.

\section{Attitude Questions}

In the introductory Sect. 1, I have given a first illustration of the non-commutative character of attitude question. In this section, I will start with summarizing the basic findings concerning order effects for attitude questions. Then I will discuss how the orthoalgebraic framework can describe the established phenomena. Finally, I will consider a recent formalization of Jung's personality theory (Blutner and Hochnadel 2010), and I will debate the explanatory value of the approach.

\subsection{Question Order Effects in Survey Research}

Schuman and Presser (1981) described two kinds of ordering effects, which they called 'consistency' and 'contrast' effects. In a more recent article, Moore (2002) reports on 


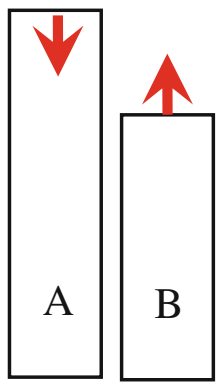

Consistency

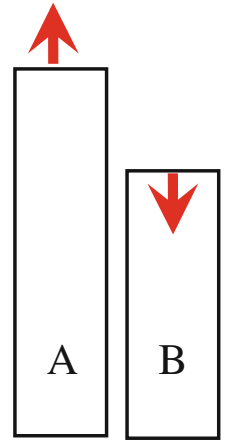

Contrast

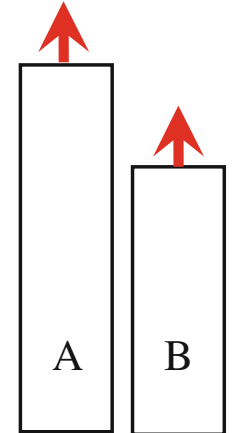

Additive

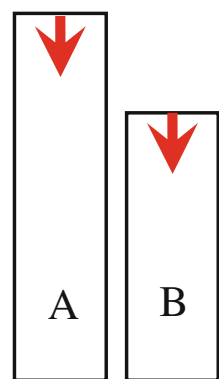

Subtractive

Fig. 11 Four types of order effects for attitude questions. The size of the blocks for questions $A$ and $\mathrm{B}$ indicates the percentage of 'yes'-answers when the questions appear isolated. The arrows indicate whether the percentage of 'yes'-answers to these questions increases or decreases if the question is preceded by the alternative question (either A or B).

Table 1 Illustration of the consistency effect (following Moore 2002)

\begin{tabular}{|c|c|c|}
\hline \multirow[t]{3}{*}{ Consistency effect } & \multicolumn{2}{|c|}{ Percentage saying "yes" } \\
\hline & \multicolumn{2}{|c|}{$\begin{array}{l}\text { Do you generally think Clinton } \\
\text { and Al Gore is honest and } \\
\text { trustworthy }\end{array}$} \\
\hline & Clinton & Al Gore \\
\hline Mentioned in isolation & 50 & 68 \\
\hline $\begin{array}{l}\text { Mentioned second (directly } \\
\text { after the other person) }\end{array}$ & 57 & 60 \\
\hline
\end{tabular}

the identification of two different types of question-order effects termed as 'additive' and 'subtractive'. Figure 11 gives a schematic sketch of the four different types of question ordering effects: Consistency can be exemplified with the case of accepted communist reporters from the introductory part. Another example is shown in the following table (adapted from Moore 2002). It does not need any comments.

Replacing the names of the prominent persons by two other prominent names can change the picture dramatically as shown in Table 2 . Here the names of two Republican leaders in the Congress are inserted, senate majority leader Robert Dole and speaker of the House Newt Gingrich.

An explanation of the differences between Tables 1 and 2 is not immediately clear. Possibly it has to do with "the symbiotic relationship of the president and vice-president, for example, versus the often competing roles of House Speaker and senate majority leader" (Moore 2002: 84). Whereas the similarities are accommodated in the first case, the differences are stressed in the second case.

Table 3 gives an illustration of the additive effect. The table shows that the amount of racial hostility among blacks/whites appears to increase when a related question about racial hostility among whites/blacks is asked before. 
Table 2 Illustration of the contrast effect (following Moore 2002)

Table 3 Illustration of the additive effect (following Moore 2002)

$$
\text { Contrast effect }
$$

Percentage saying "yes"

Do you generally think Gingrich and Dole is honest and trustworthy

\begin{tabular}{lll}
\cline { 2 - 3 } & Gingrich & Dole \\
\hline Mentioned in isolation & 41 & 60 \\
$\begin{array}{l}\text { Mentioned second (directly } \\
\text { after the other person) }\end{array}$ & 33 & 64 \\
\hline
\end{tabular}

Table 4 Illustration of the subtractive effect (following Moore 2002)

\begin{tabular}{|c|c|c|}
\hline \multirow[t]{3}{*}{ Subtractive effect } & \multicolumn{2}{|c|}{ Percentage saying "yes" } \\
\hline & \multicolumn{2}{|c|}{$\begin{array}{l}\text { As you may know, former Major League player Pete Rose and } \\
\text { Shoeless Joe Jackson is ineligible for baseball's Hall of Fame due } \\
\text { to charges that he had gambled on baseball games. Do you think he } \\
\text { should be eligible for admission to the Hall of Fame? }\end{array}$} \\
\hline & Pete Rose & Shoeless Joe Jackson \\
\hline Mentioned in isolation & 64 & 45 \\
\hline $\begin{array}{l}\text { Mentioned second (directly } \\
\text { after the other person) }\end{array}$ & 52 & 33 \\
\hline
\end{tabular}

Again, an explanation of this effect is not immediately clear. Possibly it has to do with overcoming a certain threshold in admitting racial hostility when a related question is asked before.

The final effect is subtraction as illustrated in Table 4, where two eccentric American baseball gamers are involved.

We can only speculate about the decrease of acceptance when the second question is asked. "When asked about the second ballplayer, respondents apparently became more sensitive to the larger framework issue of how many exceptions should be made for ballplayers who violate baseball's rule. For many people, it appears as though consideration of one exception, whether it was for Rose or for Jackson, made them less likely to consider a second exception.” (Moore 2002: 87). 
Table 5 Ordering effects as resulting from phase shifts $\delta$ (adopted from Wang and Busemeyer 2011)

\begin{tabular}{lllll}
\hline & $\Delta_{\mathbf{b}}(\mathbf{a})$ & $\Delta_{\mathbf{a}}(\mathbf{b})$ & Phase factor & Condition \\
\hline Assimilation & + & - & {$[[\mathbf{a} ; \mathbf{b}]]_{u}>\cos \delta>[[\mathbf{b} ; \mathbf{a}]]_{u}$} & {$[[\mathbf{a}]]_{u}>[[\mathbf{b}]]_{u}$} \\
Contrast & - & + & $\left.[[\mathbf{a} ; \mathbf{b}]]_{u}\right)<\cos \delta<[[\mathbf{b} ; \mathbf{a}]]_{u}$ & {$[[\mathbf{a}]]_{u}>[[\mathbf{b}]]_{u}$} \\
Additive & + & + & $\cos \delta<[[\mathbf{a} ; \mathbf{b}]]_{u},[[\mathbf{b} ; \mathbf{a}]]_{u}$ & None \\
Subtractive & - & - & $\cos \delta>[[\mathbf{a} ; \mathbf{b}]]_{u},[[\mathbf{b} ; \mathbf{a}]]_{u}$ & None \\
\hline
\end{tabular}

\subsection{An Orthoalgebraic Approach to Question Order Effects}

Recently, Wang and Busemeyer (2011) have shown that an orthoalgebraic approach can describe all four types of order effects. They define the ordering effect as follows (using the present notations):

$$
\left.\Delta_{\mathbf{a}}(\mathbf{b})=[[\mathbf{a} ; \mathbf{b}]]_{u}+\left[\left[\mathbf{a}^{\prime} ; \mathbf{b}\right]\right]_{u}-[[\mathbf{b}]]_{u} \text { (note: we write } \mathbf{a}^{\prime} \text { instead of } \neg \mathbf{a}\right)
$$

Hereby $[[\mathbf{a} ; \mathbf{b}]]_{u}$ is the probability of the sequence of two propositions (described by projection operators as explained in Sect. 4.3). In the classical case of commuting operators the order effects becomes zero. In the non-classical the following fact can be proven considering a pure state $u ; \delta$ is a phase shift parameter introduced by factorizing the complex number defined by the scalar product $\mathbf{a} u \cdot \mathbf{b} u$.

$$
\Delta_{\mathbf{a}}(\mathbf{b})=2\left([[\mathbf{a} ; \mathbf{b}]]_{u}-\left([[\mathbf{a}]]_{u}[[\mathbf{b}]]_{u}\right)^{1 / 2} \cos \delta\right)
$$

\section{Proof}

$$
\begin{aligned}
{[[\mathbf{a} ; \mathbf{b}]]_{u}+\left[\left[\mathbf{a}^{\prime} ; \mathbf{b}\right]\right]_{u}-[[\mathbf{b}]]_{u}=} & \mathbf{b a} u \cdot \mathbf{b a} u+\mathbf{b a}^{\prime} u \cdot \mathbf{b} \mathbf{a}^{\prime} u-\left(\mathbf{b a} u+\mathbf{b a}^{\prime} u\right) \\
& \cdot\left(\mathbf{b} \mathbf{a} u+\mathbf{b a}^{\prime} u\right) \\
= & -\mathbf{b a} u \cdot \mathbf{b} \mathbf{a}^{\prime} u-\mathbf{b a}^{\prime} u \mathbf{b a} u \\
= & -2 \operatorname{Re}\left(\mathbf{b a} u \cdot \mathbf{b} \mathbf{a}^{\prime} u\right) \\
= & -2 \operatorname{Re}(\mathbf{b a} u \cdot(\mathbf{b}-\mathbf{b a}) u) \\
= & 2 \mathbf{b a} u \cdot \mathbf{b a} u-2 \operatorname{Re}(\mathbf{a} u \cdot \mathbf{b} u) \\
= & 2\left([[\mathbf{a} ; \mathbf{b}]]_{u}-\left([[\mathbf{a}]]_{u}[[\mathbf{b}]]_{u}\right)^{1 / 2} \cos \delta\right)
\end{aligned}
$$

In dependence of the parameter $\delta$, the following order effects are defined (Table 5).

Can insights of quantum theory, initially invented to explain order effects on measurements in physics, be used for providing a natural explanation of order effects of survey questions? The previous discussion has shown that the general orthoalgebraic framework can describe all four types of question order effects. However, an explanation demands more than a description of the phenomena using a particular theoretic framework.

In discussing different approaches to decision making, Johnson and Busemeyer (2010) have considered three stages of the theoretical development:

(i) normative approach — reducing decision problems to mathematical optimization problems; 
(ii) descriptive approach-describing how humans actually make decisions;

(iii) computational approach — seeking to understand the underlying cognitive processes that produce the behavior described by the second approach.

Since the orthoalgebraic approach is a proper generalization of the classical Boolean approach, it brings the normative and the descriptive approach more closely together (Blutner 2010). However, it is not a computational theory in the sense of Johnson and Busemeyer (2010) and does not really provide an explanation of the question order effects. In contrast to quantum mechanics where we find Bohr's helpful correspondence principle, which directs the translation from classical theories into the orthoalgebraic formalism, the situation is different in the case of survey research. There is simply no formal classical theory available that could be translated into the orthoalgebraic formalism. ${ }^{22}$

In the next subsection, I will debate a further example where we find question order effects, this time pursuing the computational approach.

\subsection{Attitude Questions and C.G. Jung's Personality Theory}

I will sketch now a potential application of the present theory of attitude questions. It relates to personality theory as developed by Jung (1921). Jung developed his theory after almost 20 years of practical experience and work as a specialist in psychiatric medicine. In his book, Jung gave a careful analysis of the universals and differences of human personalities. Jung's theory is based on three psychological opposites, equally valuable but realized with different preferences for different personalities. The first opposite (a) represents the extravert/introvert opposite. This opposition reflects the most popular part of Jung's theory. We find this opposition in several theories, notably Hans Eysenck's, although it is often hidden under alternative names such as "sociability" and "surgency". Introverts are people who prefer the internal world of their own thoughts, feelings, fantasies and dreams, while extroverts prefer the external world of things, events, people and activities. ${ }^{23}$ The two other oppositions refer to what Jung

22 I should acknowledge the existence of phenomenological models that can provide interesting insights into the nature of order effects. For instance, Johnson et al. (2007), Weber and Johnson (2006, 2009) have proposed that memory processes can be used to model decision tasks. "This approach, most recently dubbed 'Query theory', assumes that preferences that drive choice and other decisions are based on a collection of serially posed queries to memory concerning relevant characteristics of the task. For example, if deciding whether to buy a certain digital camera, an individual might attempt to recall experiences with similar models or generate the pros and cons of buying the camera. Query theory is able to explain some empirical trends in human decision behavior by embellishing this simple notion with what is known about human memory, such as serial position effects, priming, and interference. Although the theory's assumptions have been empirically supported, at this point it has not been formally introduced as a mathematical model or at a specific algorithmic level, as the preceding computational models have." (Johnson and Busemeyer 2010: 745). However, this is not a kind of formal theory that has to be translated into the orthoalgebraic formalism. Rather, it is a system of informal ideas that can possibly be explicated and mathematicized in the new formalism.

23 The words have become confused with ideas like shyness and sociability, partially because introverts tend to be shy and extroverts tend to be sociable. Jung, however, intended for them to rather refer to whether an individual tends to face toward the persona and outer reality or toward the collective unconscious and its archetypes. 
Fig. 12 The four psychological functions according to Jung (1921) organized in pairs of two opposing functions. The numbers enumerate eight sectors in dependence of what are the two dominant functions (for instance, sector 1 shows feeling (F) as the primary function and intuition $(\mathrm{N})$ as the secondary function)

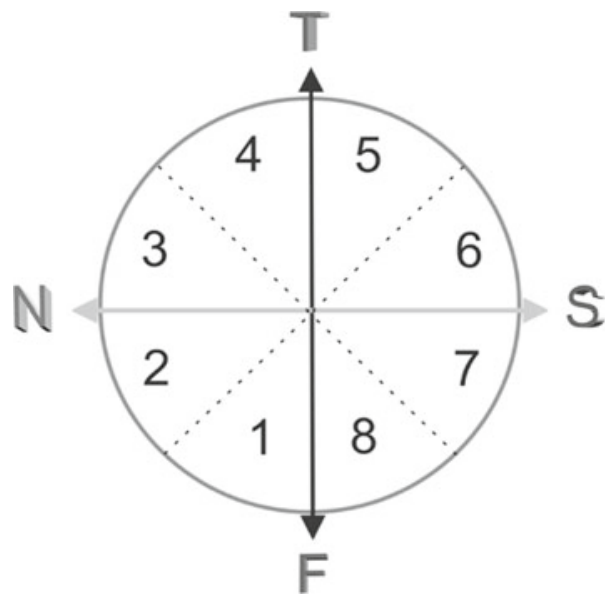

calls the four psychological functions. They consists of two opponent pairs: (i) sensing $(S)$ and intuition $(N)$-related to two opponent ways of perceiving information, either directly by the senses or in a rather indirect way by the integration of large amounts of information; (ii) thinking $(T)$ and feeling $(F)$-related to two opponent ways of judging information, either by reasoning or by evaluation.

The three opposites are exemplified by three different types of forced choice questions illustrated in (48):

(48) a. When the phone rings, do you hasten to get to it first, or do you hope someone else will answer? (E/I)

b. In order to follow other people do you need reason, or do you need trust? (T/F)

c. Are you more attracted to sensible people or imaginative people? $(\mathrm{S} / \mathrm{N})$

When a person answers with 'yes' to the first question, this answer is an indication of extraversion (E); if she answers with 'no' the answer indicates introversion (I). Similarly for (48b): here a 'yes'-answer indicates thinking (T) and a 'no'-answer indicates feeling (F). And for (48c): a 'yes'-answer indicates sensing (S) and a 'no'-answer indicates intuition $(\mathrm{N})$.

With regard to the psychological function, Jung says that we all have them. We just have them in different proportions. Each of us has a superior function, which we prefer and which is best developed in us, a secondary function, which we are aware of and use in support of our superior function. Further, we still have the two other functions, but they are less developed. In order to convey his idea of how the four functions work together, Jung offered the image of a cross. Figure 12 shows an image which is a slight modification of the original picture (Jung et al. 1968, p. 60).

Comparing Jung's drawing (Fig. 12) with the Bloch sphere (projected on the $x-z$-plane; Fig. 7a) suggests to represent the four psychological functions as Pauli spin matrices (Blutner and Hochnadel 2010). In terms of Pauli spin matrices the opponent pair $\mathrm{S} / \mathrm{N}$ is represented by $S=\sigma_{x} / N=-\sigma_{x}$ and the pair $\mathrm{T} / \mathrm{F}$ is represented by $T=\sigma_{z} / F=-\sigma_{z}$. I have to stress the point that this choice is primarily motivated 
by Jung's idea of discriminating 8 basic personality types in dependence of one of four primary psychological functions $(T, F, S, N)$ and two secondary functions that correspond to the complementary pair, either $\mathrm{S} / \mathrm{N}$ or $\mathrm{T} / \mathrm{F}$.

According to Jung (1921), the extraversion/introversion opponent pair should not be seen as an independent dimension in addition to the four psychological functions. Rather, Jung sees each psychological function realized in one particular attitude (extraversion or introversion or something in between). Hence there are no pure psychological functions. They are always in dependence of a particular attitude. I will ignore this exciting aspect of Jung's theory here and its formal treatment by entangling two qubits, one describing the psychological functions and the other describing their attitudes. Instead, the interested reader is referred to the original literature (Blutner 2010; Blutner and Hochnadel 2010) for more details and empirical judgment of the proposed theory.

\section{Conclusions}

Taking the lead from orthodox von Neumann quantum theory (Von Neumann 1932), I have introduced a handy generalization of the Boolean approach to propositions and questions: the orthoalgebraic framework. I have demonstrated that this formalism relates to a formal theory of questions (or 'observables' in the physicist's jargon). Surprisingly, this theory allows formulating conditional questions, and thus it provides the semantic power for managing question semantics. In the case of commuting observables, there are close similarities between the orthoalgebraic approach to questions and the Jäger/Hulstijn approach to question semantics. However, the present approach is more powerful. It is able to overcome most of the difficulties of the Jäger/Hulstijn approach. Further, I have demonstrated that the present approach can be seen as a decorated partition theory of questions if question order effects are excluded. Hence, it is fully compatible with the structured meaning approach to questions.

In the last part of the paper, I have discussed non-commuting operators and question order effects. I have argued that the order of the questions can definitely be important, for instance in the case of attitude questions with a flexible dimension of decision. In such situations most people do not have a predetermined opinion. Instead, the opinion is formed to a large extent during the process of questioning in a context-dependent way. This naturally leads to the question order phenomenon. I have demonstrated that the present framework provides an adequate description of the four possible question order effects discussed in the literature on survey research (Moore 2002).

The present theory should not only be of interest for scholars of formal semantics, but also for scholars from the field of quantum theory. It is clear from physics that observables are questions of some kind but till now nobody has looked at observables as real questions in natural language with their own semantics. As far as I can see, this is the first attempt where the connection is made between physical observables and theories for the semantics of questions. An important methodological issue relates to the descriptive power of a theory and its explanatory value. It could be argued that the present formalism is surely adequate for describing quantum phenomena in physics, but much too general and powerful when applied to the semantics of natural language. 
In fact, we have mainly discussed the case of commuting observables/question, i.e. we have restricted ourselves to the classical case of Boolean algebras. Why then use such a powerful formalism?

There are several aspects that can be discussed in this regard. First, there is the historical interest to relate the formal semantics of questions as developed by Groenendijk and Stokhof (1984, 1997), Krifka (2001) and others with the formal treatment of observables in quantum physics (e.g. Birkhoff and von Neumann 1936; Dalla Chiara et al. 2004; Kalmbach 1983; Piron 1976; Von Neumann 1932). One result of this comparison is the observation that quantum physics corresponds with a decorated partition theory, which has much more in common with a structured meaning approach than with the GS partition theory of questions. Second, the operator formalism of quantum mechanism allows a straightforward analysis of conditional questions. Possibly we could also implement the relevant ideas in the more traditional theory of structured meanings. However, the operator formalism seems to be very natural for a uniform treatment of questions, answers, and propositions. Third, the full orthoalgebraic framework-without the restriction to commuting observables—can be useful for understanding how quantum-like features are generated on the macro-level of cognition. I have stressed this point in Sect. 1 already and I have to stress it again at the end of this paper. The phenomena of opinion forming and the proper treatment of diagnostic questions (personality diagnostics) are typical cases in point. I have given some examples in the last section which also show the usefulness of Pauli spin operators in two-dimensional Hilbert spaces (for more discussion, see Blutner and Hochnadel 2010). The application of these specific elements of the formalism restricts the general framework considerably and leads to a series of predictions, which can be tested empirically.

There are several tasks that have to be left for future research. A first couple of tasks, for instance, are the development of the inferential machinery of the orthoalgebraic formalism, the extension to $w h$-questions, and the working out of the probabilistic component. A second important problem is a careful discussion of the normative/descriptive issue. In this connection it can be discussed whether the present orthoalgebraic framework can solve puzzles of bounded rationality (Aerts 1982; Aerts et al. 2008; Blutner 2009; Bruza et al. 2009; Franco 2007a; Khrennikov 2006). A third important problem is the proper distinction between uncertainty and ignorance, a problem that is highly relevant for any advanced theory of questions and answers. In general, ignorance is reasonable if the expected benefits of information are too small relatively to the costs. A typical situation can be found in elections, where people in general choose to remain uninformed (Downs 1957). By contrast, uncertainty refers to situations where people use statistical information to optimize their decision. Recently, it has been argued by Franco (2007b) that the behavior of people under rational ignorance can be described best within the quantum mechanics formalism, in which the states of the system are described by vectors in the Hilbert space. Alternatively, a stochastic mixture of the eigenstates of the operators under discussion represents people that reason under uncertainty. In rational-ignorance regime a kind of ignorance principle holds. It states that the product of the variances relevant to at least two questions has a non-trivial lower bound. The inventor of the uncertainty principle in quantum mechanics, Werner Heisenberg, had a fine sense for applying his principle outside the 
domain of physics. When asked about his attitude towards Christianity he used to say, with a fine irony befitting the inventor of the uncertainty principle: "If someone were to say that I had not been a Christian, he would be wrong. But if someone were to say that I had been a Christian, he would be saying too much" (quoted from Lindley 2008: 77).

If there is a bit of truth in the supposition that the abstract formalism of quantum mechanics will find useful applications in the domain of cognition, then this suggests that an active dialogue between the traditional model-theoretic approaches to semantics and the orthoalgebraic paradigm is mandatory. The use of know-how from physics and quantum information science is a very substantial aspect of the orthoalgebraic approach. It helps to integrate the different disciplines and makes it possible that formal semantics descends from its ivory tower and engages in personality diagnostics and social problems. It leaves the protected intellectual field of 'pure science' and extends formal semantics to the fruitful field of applied sciences.

Acknowledgments Thanks for valuable comments and discussions go to Maria Aloni, Anton Benz, Stefan Blutner, Hans-Martin Gärtner, Peter beim Graben, Manfred Krifka, Michiel van Lambalgen, Salvador Mascarenhas, Emar Maier, Floris Roelofsen, and two anonymous referees of JoLLI. Further, I thank the participants of an ESSLLI (2011) class on geometric models of meaning (see http://www.blutner.de/esslli), my students on quantum probabilities in Amsterdam (see http://www.blutner.de/uncert), and the participants of my lectures on ortho-algebraic semantics held at ZAS in 2008. Special thanks go to Anton Benz and Hans-Martin Gärtner for initiating the ZAS lectures.

Open Access This article is distributed under the terms of the Creative Commons Attribution License which permits any use, distribution, and reproduction in any medium, provided the original author(s) and the source are credited.

\section{References}

Aerts, D. (1982). Example of a macroscopical classical situation that violates Bell inequalities. Lettere Al Nuovo Cimento, 34(4), 107-111.

Aerts, D., Broekaert, J., \& Gabora, L. (2008). A case for applying an abstracted quantum formalism to cognition, New Ideas in Psychology. Archive reference and link: http://uk.arxiv.org/abs/quant-ph/ 0404068.

Amira, H., Coecke, B., \& Stubbe, I. (1998). How quantales emerge by introducing induction within the operational approach. Helvetica Physica Acta, 71(5), 554-572.

Arita, S., \& Kaufmann, S. (2008). The Japanese unconditional operator doose. Paper presented at the 10th symposium on logic and language (LoLa 10).

Axler, S. (1996). Linear algebra done right. New York: Springer.

Birkhoff, G., \& von Neumann, J. (1936). The logic of quantum mechanics. Annals of Mathematics, 37(4), 823-843.

Blutner, R. (2009). Concepts and bounded Rationality: An application of Niestegge's approach to conditional quantum probabilities. In L. Accardi, G. Adenier, C. Fuchs, G. Jaeger, A. Y. Khrennikov, J.-Å. Larsson, \& S. Stenholm (Eds.), Foundations of probability and physics-5 (pp. 302-310). New York: American Institute of Physics Conference Proceedings.

Blutner, R. (2010). Quantum mechanics meets cognitive science: Explanatory vs. descriptive approaches. NeuroQuantology, 8(3), 314-318.

Blutner, R., \& Hochnadel, E. (2010). Two qubits for C.G. Jung's theory of personality. Cognitive Systems Research, 11(3), 243-259.

Bruza, P., Busemeyer, J. R., \& Gabora, L. (2009). Special issue on quantum cognition. Journal of Mathematical Psychology, 53(5). 
Coecke, B., Sadrzadeh, M., \& Clark, S. (2011). Mathematical foundations for a compositional distributional model of meaning. Linguistic Analysis, 36, 345-384.

DallaChiara, M. L., Giuntini, R., \& Greechie, R. (2004). Reasoning in quantum theory: Sharp and unsharp quantum logics. Berlin, New York: Springer.

Downs, A. (1957). An economic theory of democracy. New York: Harper.

Dunn, J. M., \& Hardegree, G. M. (2001). Algebraic methods in philosophical logic. Oxford: Oxford University Press.

Einstein, A., Podolsky, B., \& Rosen, N. (1935). Can quantum-mechanical description of physical reality be considered complete?. Physical Review, 47, 777-780.

Franco, R. (2007a). The conjunction fallacy and interference effects. Arxiv preprint arXiv:0708.3948.

Franco, R. (2007b). Quantum mechanics and rational ignorance. Arxiv preprint physics/0702163.

Groenendijk, J. (2009). Inquisitive semantics: two possibilities for disjunction. In P. Bosch, D. Gabelaia, \& J. Lang, Logic, language, and computation (pp. 80-94). Berlin: Springer.

Groenendijk, J., \& Stokhof, M. (1984). Studies on the semantics of questions and the pragmatics of answers. Amsterdam: Jurriaans BV.

Groenendijk, J., \& Stokhof, M. (1997). Questions. In J. van Benthem \& A. ter Meulen (Eds.), Handbook of logic and language (pp. 1055-1124). Amsterdam: Elsevier.

Hamblin, C. L. (1973). Questions in Montague English. Foundations of Language, 10(1), 41-53.

Hausser, R. (Ed.). (1983). The syntax and semantics of English mood. Dordrecht: Reidel.

Hulstijn, J. (1997). Structured information states. Raising and resolving issues. In Proceedings of MunDial97. University of Munich.

Isaacs, J., \& Rawlins, K. (2008). Conditional questions. Journal of Semantics, 25, 269-319.

Jäger, G. (1996). Only updates. On the dynamics of the focus particle only. In Proceedings of the 10th Amsterdam Colloquium (pp. 387-405).

Johnson, E. J., Haubl, G., \& Keinan, A. (2007). Aspects of endowment: A query theory of value construction. Journal of Experimental Psychology: Learning, Memory and Cognition, 33(3), 461-473.

Johnson, J. G., \& Busemeyer, J. R. (2010). Decision making under risk and uncertainty. Wiley Interdisciplinary Reviews: Cognitive Science, 1(5), 736-749.

Jung, C. G. (1921). Psychologische Typen. Zürich: Rascher.

Jung, C. G., von Franz, M.-L., \& Henderson, J. L. (1968). Der Mensch und seine Symbole. Solothurn \& Düsseldorf: Walter.

Kalmbach, G. (1983). Orthomodular lattices. London: Academic Press.

Karttunen, L. (1977). Syntax and semantics of questions. Linguistics and Philosophy, 1(1), 3-44.

Khrennikov, A. Y. (2006). Quantum-like brain: "Interference of minds". BioSystems, 84(3), $225-241$.

Krifka, M. (2001). For a structured meaning account of questions and answers. Studia Grammatika, 52, 287-319.

Krifka, M. (2004). The semantics of questions and the focusation of answers. In C. Lee, M. Gordon, \& D. Büring (Eds.), Topic and focus: Cross-linguistic perspectives on meaning and intonation. Berlin: Springer.

Lindley, D. (2008). Uncertainty. New York: Anchor Books.

Loeser, F. (1968). Interrogativlogik. Berlin: Deutscher Verlag der Wissenschaften.

Lüders, G. (1951). Über die Zustandsänderung durch den Meßprozeß. Annalen der Physik, 8, 322-328.

Miyadera, T., \& Imai, H. (2009). No-cloning theorem on quantum logics. Available from http://arxiv. org/abs/0904.3229v3.

Moore, D. W. (2002). Measuring new types of question-order effects: Additive and subtractive. The Public Opinion Quarterly, 66(1), 80-91.

Nelken, R., \& Francez, N. (1999). The algebraic semantics of questions. Paper presented at the MOL6, the 6th meeting on mathematics of language.

Nelken, R., \& Francez, N. (2000). The algebraic semantics of interrogative NPs. Grammars, 3(2), $259-273$.

Nelken, R., \& Francez, N. (2002). Bilattices and the semantics of natural language questions. Linguistics and Philosophy, 25(1), 37-64.

Niestegge, G. (2008). An approach to quantum mechanics via conditional probabilities. Foundations of Physics, 38, 241-256.

Piron, C. (1976). Foundations of quantum physics. Reading, Mass: WA Benjamin, Inc.

Rawlins, K. (2008). (Un)conditionals: An investigation in the syntax and semantics of conditional structures. Unpublished Ph.D. thesis, UCSC. 
Rédei, M. (2009). The Birkhoff-von Neumann concept of quantum logic. In K. Engesser, D. M. Gabbay, \& D. Lehmann (Eds.), Handbook of quantum logic and quantum structures (pp. 1-22). Amsterdam: Elsevier.

Roelofsen, F., \& van Gool, S. (2010). Disjunctive questions, intonation, and highlighting. In M. Aloni, H. Bastiaanse, T. D. Jager, \& K. Schulz (Eds.), Logic, language and meaning (pp. 384-394). Berlin: Springer.

Schuman, H., \& Presser, S. (1981). Questions and answers in attitude surveys: Experiments in question form, wording, and context. New York: Academic Press.

Strang, G. (2003). Introduction to linear algebra. Cambridge: Wellesley Cambridge Press.

Sudman, S., \& Bradburn, N. M. (1982). Asking questions. San Francisco: Jossey-Bass Inc Pub.

Tichy, P. (1978). Questions, answers, and logic. American Philosphical Quaterly, 15, $275-284$.

Tourangeau, R., Rips, L. J., \& Rasinski, K. (2000). The psychology of survey response. Cambridge: Cambridge University Press.

Vedral, V. (2006). Introduction to quantum information science. New York: Oxford University Press.

Velissaratou, S. (2000). Conditional questions and which-interrogatives: Master of Logic Thesis MoL2000-01, ILLC/University of Amsterdam.

Von Neumann, J. (1932). Mathematische Grundlagen der Quantenmechanik. Transl. by R. Beyer (Princeton: Princeton University Press 1955). Heidelberg: Springer.

Von Stechow, A. (1991). Focusing and backgrounding operators. In W. Abraham (Ed.), Discourse particles (pp. 37-84). Amsterdam: John Benjamins.

Wang, Z., \& Busemeyer, J. R. (2011). Explaining and predicting question order effects using a quantum probability model. Under review.

Weber, E. U., \& Johnson, E. J. (2006). Constructing preferences from memories. In S. Lichtenstein \& P. Slovic (Eds.), The construction of preference (pp. 397-410). New York, NY: Cambridge University Press.

Weber, E. U., \& Johnson, E. J. (2009). Mindful judgement and decision making. Annual Review of Psychology, 60, 53-85.

Wiśniewski, A. (1995). The posing of questions: Logical foundations of erotetic inferences. Dordrecht: Kluwer.

Zaefferer, D. (1991). Conditionals and unconditionals. In R. Cooper, K. Mukai, \& J. Peny (Eds.), Situation theory and its applications (pp. 471-492). Stanford, Cal: CSLI. 